\title{
Computation of Free Surface Flows with a Taylor- Galerkin/Pressure-correction Algorithm
}

\author{
V. NGAMARAMVARANGGUL AND M. F. WEBSTER* \\ Institute of Non-Newtonian Fluid Mechanics, \\ Department of Computer Science, \\ University of Wales, Swansea, SA2 8PP, UK.
}

Int. J. Num. Meth. Fluids, $27^{\text {th }}$ September 1999.

\begin{abstract}
SUMMARY
A semi-implicit Taylor Galerkin/pressure-correction finite element scheme (STGFEM) is developed for problems that manifest free surfaces associated with the incompressible creeping flow of Newtonian fluids. Such problems include stick-slip and die-swell flows, both with and without a superimposed drag flow, and for plane, axisymmetric and annular systems. The numerical solutions are compared with available analytical and numerical solutions, both in the neighbourhood of singularities and elsewhere. Close correspondence in accuracy is extracted to the literature for both stick-slip and die-swell flows. Stick-slip flow is used as a precursor study to the more complex free surface calculations involved for die-swell in extrudate flow. Two different free surface techniques are reported and results are analysed with mesh refinement and varying structure.
\end{abstract}

\section{INTRODUCTION}

The focus of this paper is the investigation of a finite element time-stepping scheme based on pressure-correction in its application to free surface flows for Newtonian fluids. Intrinsic to this study is the implementation of free surface location techniques. The numerical method is based upon a semi-implicit TaylorGalerkin/pressure-correction finite element method $\left(\right.$ STGFEM) ${ }^{1,2}$ that has been successfully implemented in a variety of different flow circumstances. Specific problems considered are stick-slip and die-swell flows under creeping conditions. These flows are analysed in two dimensional plane, axisymmetric and annular coordinate systems. Annular flows are taken as pressure-driven with a superimposed drag flow, chosen as characteristic case studies relevant to the industrial process of wire-coating.

For stick-slip flow, comparison is made against the analytical solution of Richardson $^{3}$ for plane creeping flow. Many problems, described via systems of partial differential equations, display singular solutions near corners or crack tips. The region between stick and slip manifests just such a singularity. The flow behaviour in the neighbourhood of such singular points is of particular interest, where high stress concentration or sharp velocity gradients prevail. This influences the solution locally and demands a high concentration of low order elements for adequate representation. To reduce this effect, $\mathrm{Okabe}^{4}$ presented the theory of semi-radial singularity mapping, that provides for stress and strain near the singularity with bounded strain energy. Following the solution of Richardson, various numerical methods were introduced to

\footnotetext{
* Author for correspondence
} 
improve accuracy. Ingham and Kelmanson ${ }^{5}$ estimated the solution in the neighbourhood of the singularity and accelerated the rate of convergence using a singular boundary element method (SBE). Kermode et al. ${ }^{6}$ calculated the solution near a singular point using a finite element method (FEM) ${ }^{7}$ and a least-squares fitting procedure. They retained the first three terms of the singular expansion series. Georgiou et al. ${ }^{8}$ improved the solution accuracy over continuous methods in the neighbourhood of the singular point using a singular finite element method (SFEM). In a subsequent study, Georgiou et al. ${ }^{9}$ further developed the integrated singular basis function method (ISBEM) in application to stick-slip and die-swell flows. These singular function methods provide a sound basis for comparison of the quality of solutions generated by the present methodology, above and beyond that of Richardson.

Extrudate flow from a die is a special case of a stick-slip flow, where the free surface shape itself must be estimated. This is an important issue in rheology and has considerable significance to polymer processing operations in industry. Richardson ${ }^{3}$ also supplied an analytical solution for this case with integral transforms, for large surface tension under creeping flow conditions and without gravitational effects. Tanner ${ }^{10}$ has provided data from the literature on the use of several numerical schemes to compute die-swell flow, e.g. finite element, finite difference and boundary element, and comments on the better performing algorithms to estimate the position of the free surface streamline. Tanner catalogues results for swelling ratio covering axisymmetric and planar dies for Newtonian and viscoelastic flows. An asymptotic result is also quoted $^{10}$ as a simple approach for estimating practical extrudate swell calculations, where the surface tension of the extrudate is not a dominant factor.

A number of authors have employed FEM techniques for creeping die-swell flow. Using a classical FEM implementation with fine meshing, Nickell et al. ${ }^{11}$ demonstrated solutions for viscous incompressible jet and free surface flows of Newtonian fluids. Chang et al. ${ }^{12}$ studied die swell for Newtonian and viscoelastic fluids by Galerkin and collocation methods. Crochet and Keunings ${ }^{13}$ dealt with slit, circular, and annular dies for Newtonian and Maxwell fluids introducing a mixed FEM. Crochet and Keunings ${ }^{14}$ went further to show that mesh refinement, with increased concentration of elements at the singularity, has a major impact on die swell calculations. We cite Silliman and Scriven ${ }^{15}$ for their work on free surface treatment for Newtonian fluids, though their principal focus was concerned with slip (see our companion study ${ }^{16}$ ) and surface tension effects on free surface shape. Phan-Thien ${ }^{17}$ also considered slip effects with a boundary element method, in planar flows for viscoelastic fluids. This study is relevant for the proposed alternative free-surface location technique therein. Beverly and Tanner ${ }^{18}$ used boundary and finite element methods to consider extrusion of Newtonian fluids at finite Reynolds number for planar, axisymmetric and three-dimensional dies. They found that in an unconstrained extrudate the particles in the free extrudate will follow spirals or helices. In passing, we point out that thermal effects have also been found to influence free surface shape. ${ }^{19}$

Beyond the consideration of numerical solutions, some experimental results are presented in Butler and Bush $^{20}$ and Ahmed et al. ${ }^{21}$ Butler and Bush provided experimental evidence for dilute viscoelastic fluids (polyisobutylene-polybutene) in axisymmetric isothermal flows. Ahmed et al. found correspondence between experimental observations and the numerical solutions derived from a FEM, in planar entry flows and die-swell flows for molten polyethylenes.

Our interests lie in the generalisation of the STGFEM to incorporate the treatment of free surfaces and, in particular, in applications for non-Newtonian flows. In the case of planar stick-slip flow, the STGFEM approach is shown to provide accurate numerical results as compared to analytical solutions for velocity and pressure. Close correspondence is extracted for our numerical solutions near the singularity with those of the literature. The influence of die-swell is established in contrast to stick-slip 
flow. We are able to quantify the difference that drag flow has on stick-slip flow, via the change in pressure drop, peak shear rates, and adjustment in free stream velocity. Likewise, we are able to draw on comparison between die-swell and die-swell/drag flow, to indicate the reduction in swelling ratio and angle, and peak shear rates. In addition, this paper provides a useful pilot study for the analysis of annular pressuredriven drag flows, typical of those that arise in tube or pressure-tooling settings for wire-coating.

\section{GOVERNING EQUATIONS}

For Newtonian fluids and incompressible isothermal flow in the absence of body forces, the governing equations are those of generalised momentum and continuity that may be expressed as :

$$
\begin{gathered}
\rho \mathbf{U}_{\mathrm{t}}=\nabla \cdot(\mu \nabla \mathbf{U})-\rho \mathbf{U} \cdot \nabla \mathbf{U}-\nabla \mathrm{p} \\
\nabla \cdot \mathbf{U}=0
\end{gathered}
$$

where variables velocity $(\mathbf{U})$ and pressure (p) are defined over space and time with temporal derivative represented as $\left(\mathbf{U}_{\mathrm{t}}\right)$. Material parameters are given via density $(\rho)$ and viscosity $(\mu)$.

For constant $\mu$, the celebrated Navier-Stokes equations emerge. To nondimensionlise, we select the following characteristic scales: length $\mathrm{L}$, velocity $\mathrm{V}$, time $\frac{\mathrm{L}}{\mathrm{V}}$, pressure $\frac{\mu_{0} \mathrm{~V}}{\mathrm{~L}}$. We may define the following dimensionless variables and differential operators:

$$
\begin{aligned}
& \mathrm{U}^{*}=\frac{1}{\mathrm{~V}} \mathrm{U} \quad, \quad \mathrm{p}^{*}=\frac{\mathrm{L}}{\mu_{0} \mathrm{~V}} \mathrm{p} \quad, \quad \mathrm{t}^{*}=\frac{\mathrm{V}}{\mathrm{L}} \mathrm{t} \\
& \mathrm{Z}^{*}=\frac{1}{\mathrm{~L}} \mathrm{Z} \quad, \quad \mathrm{r}^{*}=\frac{1}{\mathrm{~L}} \mathrm{r} \quad, \quad \mu^{*}=\frac{1}{\mu_{0}} \mu \\
& \nabla^{*}=\mathrm{L} \nabla \quad, \quad \frac{\mathrm{D}}{\mathrm{Dt}^{*}}=\frac{\mathrm{L}}{\mathrm{V}} \frac{\mathrm{D}}{\mathrm{Dt}}
\end{aligned}
$$

where $\mu_{0}$ is a reference viscosity.

Substitution of the above dimensionless variables and differential operators into equation (1) and (2), yields the non-dimensional generalised Navies-Stokes equations, that may be stated in the following form:

$$
\begin{gathered}
\operatorname{Re} \mathbf{U}_{\mathrm{t}}=\nabla \cdot(\nabla \mathbf{U})-\operatorname{Re} \mathbf{U} \cdot \nabla \mathbf{U}-\nabla \mathrm{p} \\
\nabla \cdot \mathbf{U}=0
\end{gathered}
$$

where $\operatorname{Re}=\frac{\rho \mathrm{LV}}{\mu_{0}}$, the non-dimensional group number termed the Reynolds number.

\section{NUMERICAL SCHEME}

\subsection{Discretisation}

To solve the Navier-Stokes equations (2a), together with the incompressibility constraints (equation $2 \mathrm{~b}$ ), we employ a semi-implicit time-stepping procedure, namely a Taylor-Galerkin/pressure-correction finite element scheme ${ }^{1}$ as cite above. Briefly, the Taylor-Galerkin based algorithm is a fractional step method, that semi-discretises first 
in the temporal domain, using Taylor series expansions in time and a pressurecorrection procedure, to extract a time-stepping scheme of second-order accuracy. The discretisation is completed via a spatial Galerkin finite element method. We assume that the flow domain is discretised into a triangular mesh, and that piecewise continuous linear (pressure) and quadratic (velocity) interpolation functions apply on such elemental regions. The Taylor-Galerkin algorithm has three distinct fractional stages per time step as follows:

Stage 1 : given initial velocity and pressure fields, non-divergence-free $u^{n+\frac{1}{2}}$ and $\mathrm{u}^{*}$ fields are calculated via a two-step predictor-corrector procedure. The corresponding mass matrix governed equations are solved iteratively by a Jacobi method.

Stage 2 : using $\mathrm{u}^{*}$, calculate the pressure difference $\left(\mathrm{p}^{\mathrm{n}+1}-\mathrm{p}^{\mathrm{n}}\right)$ via a Poisson equation, applying a Choleski method of solution.

Stage 3 : using $\mathrm{u}^{*}$ and pressure difference $\left(\mathrm{p}^{\mathrm{n}+1}-\mathrm{p}^{\mathrm{n}}\right)$, determine a divergence free velocity field $\mathrm{u}^{\mathrm{n}+1}$ by Jacobi iteration.

Adopting quadratic and linear interpolations , $\mathbf{U}(\mathrm{x}, \mathrm{t})$ and $\mathrm{P}(\mathrm{x}, \mathrm{t})$, to the solution where

$$
\mathbf{U}(\mathbf{x}, \mathrm{t})=\mathrm{U}_{\mathrm{j}}(\mathrm{t}) \Phi_{\mathrm{j}}(\mathbf{x}), \mathbf{P}(\mathbf{x}, \mathrm{t})=\mathrm{P}_{\mathrm{j}}(\mathrm{t}) \psi_{\mathrm{j}}(\mathbf{x})
$$

we may proceed to solve equations (2a)-(2b). The fully discrete formulation STGFEM over a single time step, $\Delta \mathrm{t}=\mathrm{t}^{\mathrm{n}+1}-\mathrm{t}^{\mathrm{n}}$, may be represented in the following matrixvector notation :

Stage 1a

$$
\left[\frac{2 \operatorname{Re}}{\Delta \mathrm{t}} \mathbf{M}+\frac{1}{2} \mathbf{S}_{\mathrm{u}}\right]\left(\mathbf{U}^{\mathrm{n}+\frac{1}{2}}-\mathbf{U}^{\mathrm{n}}\right)=\left\{-\left[\mathbf{S}_{\mathrm{u}} \mathbf{U}+\operatorname{Re} \mathbf{N}(\mathbf{U}) \mathbf{U}\right]+\mathbf{L}^{\mathrm{T}} \mathbf{P}\right\}^{\mathrm{n}}
$$

Stage $1 b$

$$
\left[\frac{\mathrm{Re}}{\Delta \mathrm{t}} \mathbf{M}+\frac{1}{2} \mathbf{S}_{\mathrm{u}}\right]\left(\mathbf{U}^{*}-\mathbf{U}^{\mathrm{n}}\right)=\left[-\mathbf{S}_{\mathrm{u}} \mathbf{U}+\mathbf{L}^{\mathrm{T}} \mathbf{P}\right]^{\mathrm{n}}-\operatorname{Re}[\mathbf{N}(\mathbf{U}) \mathbf{U}]^{\mathrm{n}+\frac{1}{2}}
$$

Stage 2

$$
\mathbf{K}\left(\mathbf{P}^{\mathrm{n}+1}-\mathbf{P}^{\mathrm{n}}\right)=-\frac{2}{\Delta \mathrm{t}} \mathbf{L} \mathbf{U}^{*}
$$

Stage 3

$$
\frac{\mathrm{Re}}{\Delta \mathrm{t}} \mathbf{M}\left(\mathbf{U}^{\mathrm{n}+1}-\mathbf{U}^{*}\right)=\frac{1}{2} \mathbf{L}^{\mathrm{T}}\left(\mathbf{P}^{\mathrm{n}+1}-\mathbf{P}^{\mathrm{n}}\right)
$$

where variables are defined as: nodal vectors at time $\mathrm{t}^{\mathrm{n}}$ for velocity $\left(\mathbf{U}^{\mathrm{n}}\right)$ and pressure $\left(\mathbf{P}^{\mathrm{n}}\right)$, an intermediate non-solenoidal nodal velocity vector $\left(\mathbf{U}^{*}\right)$, mass matrix $(\mathbf{M})$, momentum diffusion matrix $\left(\mathbf{S}_{\mathrm{u}}\right)$, a pressure stiffness matrix $(\mathbf{K})$, convection matrix $(\mathbf{N}(\mathbf{U}))$ and divergence/pressure gradient matrix $(\mathbf{L})$.

In matrix notation, we have

$$
\begin{gathered}
\mathbf{M}_{\mathrm{ij}}=\int_{\Omega} \mathrm{r} \phi_{\mathrm{i}} \phi_{\mathrm{j}} \mathrm{d} \Omega \\
\mathbf{K}_{\mathrm{ij}}=\int_{\Omega} \mathrm{r}\left(\nabla \psi_{\mathrm{i}} \cdot \nabla \psi_{\mathrm{j}}\right) \mathrm{d} \Omega \\
\mathbf{N}(\mathbf{U})_{\mathrm{ij}}=\int_{\Omega} \mathrm{r}_{\mathrm{i}}\left(\mathrm{U}_{\mathrm{k}}^{1} \phi_{1} \frac{\partial \phi_{\mathrm{j}}}{\partial \mathrm{x}_{\mathrm{k}}}\right) \mathrm{d} \Omega \\
\left(\mathbf{L}_{\mathrm{k}_{\mathrm{ij}}}=\int_{\Omega} \mathrm{r} \phi_{\mathrm{i}} \frac{\partial \phi_{\mathrm{j}}}{\partial \mathrm{x}_{\mathrm{k}}} \mathrm{d} \Omega\right.
\end{gathered}
$$




$$
\begin{array}{cc}
\left(\mathbf{S}_{\mathrm{u}}\right)_{\mathrm{ij}}=\left(\mathbf{S}_{l \mathrm{~m}}+\mathbf{V}_{\mathrm{lm}}\right)_{\mathrm{ij}} & \\
\left(\mathbf{S}_{\operatorname{lm}}\right)_{\mathrm{ij}}=\int_{\Omega} \mathrm{r} \mu\left(\chi_{\mathrm{lk}} \frac{\partial \phi_{\mathrm{i}}}{\partial \mathrm{x}_{\mathrm{k}}} \frac{\partial \phi_{\mathrm{j}}}{\partial \mathrm{x}_{\mathrm{k}}}\right) \mathrm{d} \Omega, & \text { if } \mathrm{l}=\mathrm{m} \\
\left(\mathbf{S}_{\operatorname{lm}}\right)_{\mathrm{ij}}=\int_{\Omega} \mathrm{r} \mu\left(\frac{\partial \phi_{\mathrm{i}}}{\partial \mathrm{x}_{\mathrm{m}}} \frac{\partial \phi_{\mathrm{j}}}{\partial \mathrm{x}_{\mathrm{l}}}\right) \mathrm{d} \Omega, & \text { if } \mathrm{l} \neq \mathrm{m}
\end{array}
$$

where $\mathrm{k}, 1=1,2$

and $\quad \mathrm{x}_{1}=\mathrm{r}, \mathrm{x}_{2}=\mathrm{z}$

$$
\begin{array}{ll}
\chi_{\mathrm{lk}}=2, \text { if } \mathrm{l}=\mathrm{k} & \text { and } \quad \chi_{\mathrm{lk}}=1, \text { if } \mathrm{l} \neq \mathrm{k} \\
\left(\mathrm{V}_{\mathrm{lm}}\right)_{\mathrm{ij}}=\frac{2 \phi_{\mathrm{i}} \phi_{\mathrm{j}}}{\mathrm{r}^{2}}, \text { if } \mathrm{l}=\mathrm{m}=1 & \text { and }\left(\mathrm{V}_{\mathrm{lm}}\right)_{\mathrm{ij}}=0 \text { if } 1, \mathrm{~m} \neq 1
\end{array}
$$

The time-stepping procedure is monitored for convergence to a steady state via relative increment norms (using both maximum and least squares measures) subject to satisfaction of a suitable tolerance criteria, here taken as $10^{-5}$.

\subsection{Free surface location}

The extent of extrudate swell in a die-swell flow may be determined by implementing a free surface location method via a modified iterative technique (for example, in industrial casting processes). According to Crochet et al. ${ }^{22}$, the following three boundary conditions may be defined on a free surface:

$$
\begin{gathered}
\mathrm{v}_{\mathrm{r}} \mathrm{n}_{\mathrm{r}}+\mathrm{v}_{\mathrm{z}} \mathrm{n}_{\mathrm{z}}=0 \\
\mathrm{t}_{\mathrm{r}} \mathrm{n}_{\mathrm{r}}+\mathrm{t}_{\mathrm{z}} \mathrm{n}_{\mathrm{z}}=\mathrm{S}\left(\frac{1}{\rho_{1}}+\frac{1}{\rho_{2}}\right) \\
\mathrm{t}_{\mathrm{r}} \mathrm{n}_{\mathrm{z}}-\mathrm{t}_{\mathrm{z}} \mathrm{n}_{\mathrm{r}}=0
\end{gathered}
$$

with variables specification of radial velocity $\left(\mathrm{v}_{\mathrm{r}}\right)$, axial velocity $\left(\mathrm{v}_{\mathrm{z}}\right)$, components of the unit normal to the free surface $\left(n_{r}, n_{z}\right)$, surface force normal to the surface $\left(t_{r}, t_{z}\right)$, principal radii of curvature $\left(\rho_{1}, \rho_{2}\right)$ and surface tension coefficient (S).

Typically, when modelling a free surface iteratively, conditions (4) and (5) are enforced as boundary conditions. Then the normal velocity is calculated using equation (3) and this is used to describe the shape of the upper extrudate boundary for say dieswell flow, as illustrated in figure 1c. In the free jet flow the distance from the axis of symmetry is

$$
\mathrm{r}(\mathrm{z})=\mathrm{R}+\int_{\mathrm{z}=0}^{\infty} \mathrm{dz} \frac{\mathrm{v}_{\mathrm{r}}(\mathrm{z})}{\mathrm{V}_{\mathrm{z}}(\mathrm{z})}
$$

where $\mathrm{R}$ is the tube radius.

In this paper, the integral in equation (6) is evaluated by Simpsons quadrature rule, thus providing an estimate of the extrudate shape. The comparison of Richardson's ${ }^{3}$ asymptotic results for swell ratio $\left(\chi=\frac{\mathrm{R}_{\mathrm{j}}}{\mathrm{R}}, \mathrm{R}_{\mathrm{j}}\right.$ is jet radius, $\mathrm{R}$ is tube radius) with those from a finite element calculation is catalogued in Silliman and Scriven. ${ }^{15}$ Phan-Thien ${ }^{17}$ focuses on the extrudate shape as it varies due to slip at the wall and compares the swelling ratio for various critical wall shear stresses, employing an alternative free surface updating strategy. The implementation of the process is straightforward. First, the free surface must be estimated from a previous solution. The function describing any free surface, at time $t$ is defined as $\mathbf{h}=h(z, t)$ so that at the free surface, the following equation holds and must be updated at each time step 


$$
\frac{\partial \mathbf{h}}{\partial \mathrm{t}}=\mathrm{v}_{\mathrm{r}}-\mathrm{v}_{\mathrm{z}}\left(\frac{\partial \mathbf{h}}{\partial \mathrm{z}}\right)=\mathrm{G}(\mathbf{U}(\mathrm{t}), \mathbf{h}(\mathrm{t}))
$$

where $\mathbf{U}=\left(\mathrm{v}_{\mathrm{r}}, \mathrm{v}_{\mathrm{z}}\right)$. The free surface equation (7) is updated in time, by either a first or second-order scheme. Considered in a pointwise manner in space, the first-order Euler scheme with chosen time step $\Delta \mathrm{t}$, is provided by:

$$
\mathbf{h}(\mathrm{z}, \mathrm{t}+\Delta \mathrm{t})=\mathbf{h}(\mathrm{z}, \mathrm{t})+\Delta \mathrm{t} \mathrm{G}(\mathbf{U}(\mathrm{t}), \mathbf{h}(\mathrm{z}, \mathrm{t})) .
$$

To derive a second-order scheme, the temporal series is pursued to higher order terms :

$$
\mathbf{h}(\mathrm{z}, \mathrm{t}+\Delta \mathrm{t})=\mathbf{h}(\mathrm{z}, \mathrm{t})+\Delta \mathrm{t} \mathrm{G}(\mathbf{U}(\mathrm{t}), \mathbf{h}(\mathrm{z}, \mathrm{t}))+\frac{1}{2}(\Delta \mathrm{t})^{2} \frac{\partial}{\partial \mathrm{t}} \mathrm{G}(\mathbf{U}(\mathrm{t}), \mathbf{h}(\mathrm{z}, \mathrm{t})) .
$$

An alternative second-order scheme is a two-step implementation due to Heun,

$$
\begin{gathered}
\mathbf{h}^{\prime}=\mathbf{h}(\mathrm{z}, \mathrm{t})+\Delta \mathrm{tG}(\mathbf{U}(\mathrm{t}), \mathbf{h}(\mathrm{z}, \mathrm{t})), \\
\mathbf{h}(\mathrm{z}, \mathrm{t}+\Delta \mathrm{t})=\mathbf{h}(\mathrm{z}, \mathrm{t})+\frac{1}{2} \Delta \mathrm{t}\left[\mathrm{G}(\mathbf{U}(\mathrm{t}), \mathbf{h}(\mathrm{z}, \mathrm{t}))+\mathrm{G}\left(\mathbf{U}(\mathrm{t}), \mathbf{h}^{\prime}(\mathrm{z}, \mathrm{t})\right)\right] .
\end{gathered}
$$

The results from implementation of Euler and Heun schemes prove remarkably similar, and therefore only those for the Euler scheme are discussed in this article.

\section{PROBLEM SPECIFICATION}

There are essentially two types of problems studied here, stick-slip flow and die-swell flow. A variant within each category is to consider, in addition, a drag flow component. Poiseuille stick-slip flow is taken within a Cartesian framework, and also under an annular configuration when drag flow is imposed simultaneously. For the case of Poiseuille die-swell, the benchmark axisymmetric setting is taken first, this being followed by an annular instance with drag-flow. A visual schemata of the boundary conditions for the stick-slip flow, stick-slip/drag flow and die-swell flow are given in figure 1. Velocity conditions are imposed as essential conditions, whilst stress conditions arise naturally in weak form (see Silliman and Scriven ${ }^{15}$ ). Initial conditions for this time-stepping scheme are taken as either quiescent for stick-slip instances, or for die-swell flows, from a precomputed steady-state solution with an estimated freesurface location.

\subsection{Planar stick-slip flow}

The stick-slip flow problem consists of two regions with distinct boundary conditions, a channel section and a free jet flow section. Considering the planar case, stick or no-slip, boundary conditions apply at the channel walls, to adjust subsequently to slip boundary conditions beyond the channel, as shown in figure 1a. This implies that tangential velocity and shear stress vanish on the free surface, as does cross-stream velocity and normal stress (Cauchy stress defined as $\sigma$ ) at the outlet.

We use the notation, PS, to imply Poiseuille flow, as given by a onedimensional velocity profile of the dimensionless form $V_{x}(y)=V_{\max }\left(1-y^{2}\right)$, with maximum inlet velocity $\mathrm{V}_{\max }$. Characteristic scales of length and velocity are adopted of half channel width and average inlet velocity, respectively. This problem is solved using the STGFEM above on three uniform and one biased mesh, the details of which are specified in table 1 and illustrated in figure 2. The smallest element in the biased case is located adjacent to the singularity. Comparison of the results obtained is made against those of Richardson ${ }^{3}$ and Nickell et al. ${ }^{11}$ in section 5.1. To this end, the implementation is considered for creeping flow in the upper half plane through symmetry. A vanishing pressure datum is set on the top slip surface and outlet 
boundary. Dimensionless quantities are taken as: $\mathrm{x}_{1}=-2, \mathrm{x}_{2}=2, \mathrm{y}_{0}=0, \mathrm{y}_{1}=1$, and $\mathrm{V}_{\max }=1.5$ units.

Table 1 Finite element meshes for stick-slip flow

\begin{tabular}{|l|c|c|c|}
\hline \multicolumn{1}{|c|}{ Mesh } & Element & Total Element & $\begin{array}{c}\text { Size of element } \\
(\Delta \mathrm{h})\end{array}$ \\
\hline (a) Coarse mesh & $5 \times 20$ & 200 & 0.200 \\
\hline (b) Medium mesh & $10 \times 40$ & 800 & 0.100 \\
\hline (c) Fine mesh & $15 \times 60$ & 1800 & 0.067 \\
\hline (c) Biased fine mesh & $15 \times 60$ & 1800 & 0.024 \\
\hline
\end{tabular}

\subsection{Axisymmetric stick-slip flow (ASSF)}

The boundary conditions for the axisymmetric case of stick-slip flow are similar to those for the planar stick-slip flow described in section 4.1, figure 1a. The only difference in the governing conditions lies in the introduction of a cylindrical coordinate system. A Poiseuille flow is imposed at inlet. Advantage may be taken of symmetry radially, so that solutions are sought in the top half-plane, noting that this implies a lower symmetry boundary where the radial velocity vanishes. Characteristic scales of length and velocity are taken as channel width and maximum inlet velocity. Dimensionless quantities result of channel radius and length of unity, jet length of two. Henceforth, for all flows considered a finite small value of Reynolds number is assumed to emulate practical creeping conditions, $\mathrm{Re}=10^{-4}$. For this case, we have generated a biased fine mesh for adequate resolution, which is finer than that employed for the planar counterpart problem, with elements $18 \times 54$, nodes 4033 , and size of element $2.6083 \times 10^{-2}$, as demonstrated in figure $8 \mathrm{a}$.

\subsection{Stick-slip/drag flow (SSDF)}

This is a more complex annular flow configuration than conventional axisymmetric stick-slip flow, for which the mesh of figure $8 \mathrm{a}$ is employed. Such a problem instance is initiated from an inlet annular pressure-driven base flow with a superimposed drag flow on the inner boundary. Remaining boundary conditions follow stick-slip flow, as cited above. A schematic illustration is provided with boundary conditions in figure $1 \mathrm{~b}$. The velocity $\mathrm{v}_{\mathrm{z}}$ at the inlet is defined by equation (A.1) of the appendix. Such a specification may be found in wire-coating for example, where the inner boundary represents a wire moving at a constant speed, taken here of nondimensional radial dimension $\mathrm{a}=0.15$ units. Characteristic scales are taken for length as inlet hydraulic radius $\mathrm{R}$ and for velocity as in section 4.2 for axisymmetric stick-slip flow. This leads to equivalent flow rates in both flow settings. Dimensionless quantities result as: $\mathrm{z}_{1}=-1, \mathrm{z}_{2}=2$, jet length of 2 , wire speed $\mathrm{V}_{\text {wire }}=0.5$, and $\mathrm{b}=1.15$ units.

\subsection{Die-swell flow}

The die swell problem may be identified via two regions of different character, the shear flow within the die and the free jet flow beyond it. Each region has its unique set of boundary conditions and the problem is posed in an axisymmetric frame of reference. Poiseuille flow is imposed at the inlet. The outer wall boundary experiences stick conditions in the die section, changing to slip conditions at the free meniscus surface beyond the die. Channel radius and maximum inlet velocity are taken as 
characteristic scales, following section 4.2. A schematic representation of the problem is presented in figure 1c, with notation for Cauchy stress $(\sigma)$, unit normal vector (n) and unit tangential vector (s) to the respective surface.

Under the assumption of negligible surface tension, die-swell flow is simulated for a range of refined meshes, coarse, medium and fine, with two different mesh structures. This permits an analysis of consistency, order of accuracy and provides insight as to the influence of mesh structure on the solution. The meshing details are given in figure 10 , where $\Delta \mathrm{h}$ is a measure of the smallest size of element.

Table 2 Finite element meshes for die-swell flow

\begin{tabular}{|l|c|c|c|c|}
\hline \multicolumn{1}{|c|}{ Mesh } & Element & Total Element & Direction & $\Delta \mathrm{h}^{*} 10^{-2}$ \\
\hline (a) Coarse UD mesh & $6 \times 18$ & 216 & & 6.4550 \\
\hline (b) Medium UD mesh & $12 \times 36$ & 864 & & 3.0533 \\
\hline (c) Fine UD mesh & $18 \times 54$ & 1944 & $\square$ & 1.9667 \\
\hline (d) Coarse DU mesh & $6 \times 18$ & 216 & $\square$ & 6.4550 \\
\hline (e) Medium DU mesh & $12 \times 36$ & 864 & $\square$ & 3.0533 \\
\hline (f) Fine DU mesh & $18 \times 54$ & 1944 & $\square$ & 1.9667 \\
\hline
\end{tabular}

\subsection{Die-swell/drag flow}

This problem is a combination of those stated previously, taking the drag flow described under stick-slip with the die-swell specification. The free surface conditions remain unchanged, and the inner boundary (wire) moves at a constant speed of 0.5 units. The same characteristic scales of length and velocity, and dimensionless quantities of section 4.3 are adopted in this case. The inlet profile is determined from equation (A.1) of the appendix. This problem is simulated on the same three levels of mesh refinement as for the die-swell problem, where we have pre-selected the better performing mesh option with UD structure, see table 2, figure 10 and comments below.

\section{RESULTS AND DISCUSSION}

\subsection{Planar stick-slip flow}

First, the coarse, medium and fine meshes of table 1 and figure 2 are considered. The location of the stick-slip singularity is indicated by arrow in Figure 2 . On the medium mesh, the velocity vector plot of figure $3 \mathrm{a}$ illustrates the general pattern of the flow for the upper half plane, that is visually identical for meshes (a), (b), (c) of table 1 . This shows an initial Poiseuille flow that gradually adjusts to a plug flow. Figures $3 b$ and $3 c$ represent the horizontal $\left(V_{x}\right)$ and vertical $\left(V_{y}\right)$ velocity component line contours. Figure $3 b$ shows $V_{x}$, with no-slip at the upper boundary to channel exit, whereupon $\mathrm{V}_{\mathrm{x}}$ gradually increases, becoming faster with increasing distance along the top surface (reflecting slip conditions). The vertical velocity $\left(\mathrm{V}_{\mathrm{y}}\right)$ line plot of figure $3 \mathrm{c}$ vanishes at inlet and outlet, top surface and symmetry axis, and displays closed contours of constant value in the neighbourhood of the singularity, see Nickell. ${ }^{11}$ The centre of the plot demonstrates a peak maximum value of 0.17 units.

The shear rate $\mathrm{I}_{2}$ contour plot (figure $4 \mathrm{~b}$, representing the second invariant of the rate of strain tensor) demonstrates the formation of a singularity at the die exit, which is again represented clearly in the line plot of figure 4c. The shear rate at the top surface (figure $4 \mathrm{c})$ increases exponentially towards the die exit $(x=0)$ to a maximum of 8.28 
units. On moving away from the die, a sharp drop is displayed with shear rate tending to zero at $\mathrm{x}=2$ units. Figure $4 \mathrm{a}$ shows a contour plot of pressure for this problem. A maximum value in pressure is observed of 6.89 units at the inlet boundary, which represents the pressure drop across the flow; a minimum pressure of -2.79 units occurs near the singularity.

Table 3 gives the comparison of maximum shear rate $I_{2}$ which occurs at the top free surface, and for pressure $\mathrm{P}$ throughout the domain, for the three levels of mesh refinement. The maximum value of shear rate occurs at the singular die exit point and doubles from coarse to fine mesh solutions. The maximum value of $\mathrm{P}$ represents the pressure drop across the flow and is fairly stable around 6.75 units. Minimum values of $\mathrm{P}$ correspond to pressure pockets adjacent to the die exit within the jet flow.

Table 3 Shear rate and pressure for planar stick-slip flow: various meshes

\begin{tabular}{|cc|c|c|c|}
\hline \multicolumn{2}{|c|}{ Solution } & Course Mesh & Medium Mesh & Fine Mesh \\
\hline $\mathrm{I}_{2}$ & $\max$ & 5.86 & 8.28 & 10.14 \\
\hline $\mathrm{P}$ & $\min$ & -1.35 & -2.79 & -3.91 \\
& $\max$ & 6.76 & 6.89 & 6.75 \\
\hline
\end{tabular}

Turning to comparison against analytical solutions, we consider flow profiles for velocity and pressure. The velocity profile for $\mathrm{x} \leq 0$ (figure 5a) shows a parabolic flow that gradually flattens. Similarly for $x \geq 0$, the velocity profile of figure $5 b$ reveals an initial flattened parabolic form, which gradually adjusts to a linear pattern with increasing $\mathrm{x}$. Table 4 and figure 6 , provide tabular and graphical comparisons of velocity results with STGFEM scheme on coarse, medium and fine meshes, against the analytical solution of Richardson. The analytical solution for the streamwise velocity component $\mathrm{V}_{\mathrm{x}}$ was derived from the stream function, as identified via the formula of Richardson $^{3}$ provided in the appendix. Table 5 and figure $7 \mathrm{a}$, provide equivalent data for pressure, where the Richardson solution has been reproduced based on the graphical information recorded in Reference 3. The error in the results decreases consistently and proportionally with mesh refinement over coarse, medium and fine meshes.

Table 4 Analytical and computed velocity along axis of symmetry: various meshes

\begin{tabular}{|c|c|c|c|c|}
\hline $\mathrm{x}$ axis & Richardson & $\begin{array}{c}\text { coarse mesh } \\
\text { 200 elements }\end{array}$ & $\begin{array}{c}\text { medium mesh } \\
800 \text { elements }\end{array}$ & $\begin{array}{c}\text { fine mesh } \\
1800 \text { elements }\end{array}$ \\
\hline-1.0 & 1.4964 & 1.4956 & 1.4958 & 1.4959 \\
\hline-0.8 & 1.4899 & 1.4889 & 1.4890 & 1.4892 \\
\hline-0.6 & 1.4758 & 1.4751 & 1.4747 & 1.4749 \\
\hline-0.4 & 1.4484 & 1.4496 & 1.4479 & 1.4479 \\
\hline-0.2 & 1.4027 & 1.4086 & 1.4042 & 1.4035 \\
\hline 0.2 & 1.2798 & 1.2866 & 1.2737 & 1.2701 \\
\hline 0.4 & 1.1967 & 1.2229 & 1.2059 & 1.2006 \\
\hline 0.6 & 1.1308 & 1.1698 & 1.1494 & 1.1403 \\
\hline 0.8 & 1.0834 & 1.1303 & 1.1074 & 1.0996 \\
\hline 1.0 & 1.0516 & 1.1032 & 1.0787 & 1.0702 \\
\hline
\end{tabular}

Table 5 Analytical and computed pressure along axis of symmetry: various meshes 


\begin{tabular}{|c|c|c|c|c|}
\hline $\mathrm{x}$ axis & $\begin{array}{c}\text { Richardson } \\
\pm 0.0001\end{array}$ & $\begin{array}{c}\text { coarse mesh } \\
\text { 200 elements }\end{array}$ & $\begin{array}{c}\text { medium mesh } \\
800 \text { elements }\end{array}$ & $\begin{array}{c}\text { fine mesh } \\
1800 \text { elements }\end{array}$ \\
\hline-1.6 & 5.7264 & 5.5620 & 5.5786 & 5.5924 \\
\hline-1.2 & 4.4627 & 4.3587 & 4.3750 & 4.3887 \\
\hline-0.8 & 3.2488 & 3.1542 & 3.1688 & 3.1818 \\
\hline-0.4 & 2.0299 & 1.9834 & 1.9923 & 2.0003 \\
\hline 0.0 & 1.0348 & 0.9916 & 0.9924 & 0.9980 \\
\hline 0.4 & 0.4179 & 0.3759 & 0.3781 & 0.3801 \\
\hline 0.8 & 0.1393 & 0.1174 & 0.1203 & 0.1208 \\
\hline 1.2 & 0.0398 & 0.0354 & 0.0353 & 0.0354 \\
\hline 1.6 & 0.0149 & 0.0095 & 0.0094 & 0.0094 \\
\hline 2.0 & 0.0000 & 0.0000 & 0.0000 & 0.0000 \\
\hline
\end{tabular}

Figure $7 \mathrm{a}$ shows the variation in pressure along the centreline in contrast to the Richardson analytical solution, and those on coarse, medium and fine meshes. All predictions show consistency and close correspondence for pressure to the analytical solution (see table 6), decreasing linearly within the die, becoming more parabolic in shape in the jet region, as pressure tends to vanish. The corresponding results on accuracy for velocity and pressure are illustrated in table 6 with the comparison based on maximum error norm measure. The trend of behaviour for velocity with mesh refinement is displayed in figure $7 \mathrm{c}$, which indicates $\mathrm{O}\left(\mathrm{h}^{1.9}\right)$ inside and $\mathrm{O}\left(\mathrm{h}^{1.0}\right)$ beyond the die. Hence, the velocity solution displays almost second-order accuracy in the die flow and first-order beyond. For the fine mesh results of table 6, the error detected in velocity increases from 0.06 percent within the die to 1.77 percent beyond. For pressure, the error degradation is far less dramatic and the jet flow solution displays slightly less error than is the case for the die flow. Note, the solution scaling in error norms for pressure is taken as unity for the jet flow as the size of the solution is less than unity. The error is 2.34 percent within the die. Beyond the die, the error is represented as 3.78 percent.

Table $6 \mathrm{~L}_{\infty}$ error for velocity and pressure against analytical solution: various meshes

\begin{tabular}{|c|c|c|c|}
\hline $\mathrm{L}_{\infty}$ error & $\begin{array}{c}\text { Coarse Mesh } \\
\Delta \mathrm{h}=0.20\end{array}$ & $\begin{array}{c}\text { Medium Mesh } \\
\Delta \mathrm{h}=0.10\end{array}$ & $\begin{array}{c}\text { Fine Mesh } \\
\Delta \mathrm{h}=0.07\end{array}$ \\
\hline velocity (in die) & 0.004175 & 0.001102 & 0.000595 \\
\hline velocity (beyond die) & 0.049145 & 0.025848 & 0.017731 \\
\hline pressure (in die) & 0.028702 & 0.025803 & 0.023395 \\
\hline pressure (beyond die) & 0.041986 & 0.039850 & 0.037820 \\
\hline
\end{tabular}

In table 7 , the STGFEM velocity results (at $\mathrm{x}=0.2$ units on the free surface after the die exit) of the three mesh refinements are compared with the analytical solution of Richardson, the numerical SBE results of Ingham and Kelmanson, and the SFEM and ISBFM results of Georgiou et al. (recorded to precision quoted in original references, correcting for the noted anomaly cited in Georgiou et al. ${ }^{8}$ of Richardson's result). The STGFEM is found to be consistent across meshes, providing a convergent trend in velocity with mesh refinement. The velocity on the finest mesh lies between the analytical result of Reference 3 and the numerical results of Reference 5, 8, 9, falling within an error of about 3 percent. The corresponding figure $7 b$ shows the velocity adjustment with increasing $\mathrm{x}$ near the die exit, as the fluid travels away from the 
singularity $(\mathrm{x}=0)$. This figure also compares the analytical solution with others from the literature. From this plot and the values of table 7, we note for uniform meshing velocity agreement in trend along the top surface with Ingham et al. ${ }^{5}$ and Georgiou et al., 8 though slightly overestimated in value. The departure from the analytical solution in the results of Reference 5,8,9 may be somewhat attributed to the overall uniformity in meshing they adopt. For the biased fine meshing option, there is an increased tendency towards the analytical solution of Richardson, that reflects the improvement to be had with such an approach. At $\mathrm{x}=0.2$, the difference from the Richardson solution drops to $\mathrm{O}(.1 \%)$.

Table 7 Analytical and computed velocity results at $x=0.2$ on free surface after die exit

\begin{tabular}{|l|c|}
\hline Method & Velocity \\
\hline Analytical $^{3}$ & 0.618040 \\
\hline SBE $^{5}$ & 0.572608 \\
\hline SFEM $^{8}$ & 0.571896 \\
\hline ISBFM $^{9}$ & 0.571259 \\
\hline STGFEM (coarse mesh) & 0.690559 \\
\hline STGFEM (medium mesh) & 0.643575 \\
\hline STGFEM (fine mesh) & 0.625190 \\
\hline STGFEM (biased fine mesh) & 0.619786 \\
\hline
\end{tabular}

\subsection{Axisymmetric stick-slip flow and stick-slip/drag flow}

For both axisymmetric stick-slip flow (ASSF) and stick-slip/drag flow (SSDF) a fine mesh of figure $8 \mathrm{a}$ is used. Comparisons between these two flows for values of shear rate $\mathrm{I}_{2}$, and pressure $\mathrm{P}$ are evident in table 8 at the same level of entry flow rate.

Table 8 Axisymmetric stick-slip and stick-slip/drag flow: shear rate and pressure

\begin{tabular}{|c|c|c|c|}
\hline Solut & & ASSF & SSDF \\
\hline $\mathrm{I}_{2}$ & $\max$ & 7.93 & 6.28 \\
\hline & $\min$ & -3.92 & -3.01 \\
\hline & $\max$ & 4.88 & 4.02 \\
\hline
\end{tabular}

The velocity vector plot for stick-slip/drag flow is displayed in figure $8 \mathrm{~b}$, that reveals an initial annular flow, adjusting rapidly at the pipe outlet, to finally assume a plug flow. The radial and axial velocity line contour plots for both cases are virtually identical to the case of planar stick-slip flow and are not repeated for conciseness (see table 8 for relevant quantities). Figure $8 \mathrm{c}$ shows the pressure line contour plot, for which the initial inlet maximum value of 4.02 units decreases in a linear fashion whilst approaching the singularity, where a minimum pressure of -3.01 units is observed. The shear rate contours of figure 8d, increase in value at the top surface, reaching a peak shear rate of 6.3 units at the singular point, after which the shear rate drops sharply to zero. This is due to the dependence of shear rate upon the velocity gradient, that increases sharply in the neighbourhood of the die-exit location. The general trends of behaviour in velocity, pressure and shear rate are exposed more starkly by direct 
comparison between those for pure stick-slip flow (ASSF) and those for stick-slip/drag flow (SSDF).

The comments above are borne out by the line plots of figure 9. A comparison of axial velocity $\mathrm{V}_{\mathrm{z}}$ along the free top surface for the two flows, ASSF and SSDF, is shown in figure $9 \mathrm{a}$. The final value of free stream velocity is reduced by twenty one percent in the drag flow case, due to the influence of the moving wire on the deformation. Comparisons of pressure and $\mathrm{I}_{2}$ for ASSF and SSDF in the axial direction is made in figure $9 \mathrm{~b}$ and $9 \mathrm{c}$, respectively. The change in pressure drop between these two flows of figure $9 \mathrm{~b}$ is 17.6 percent, with an SSDF value of 4.02 units and an ASSF value of 4.88 units. Hence, drag flow imposition gives rise to a decline in the rate of pressure drop as one might expect. Patterns are similar to the planar stick-slip case of figure 7a. The shear rate profiles of figure $9 \mathrm{c}$ follow the general form of figure $4 \mathrm{c}$ for planar stick-slip flow. The behaviour of $I_{2}$ in the neighbourhood of the singularity is exposed, see also table 8 . Here, field patterns are similar in figure $8 \mathrm{~d}$ to those of figure $4 \mathrm{~b}$. The SSDF value of 6.28 units represents a reduction of twenty one percent from the ASSF value of 7.93 units. Clearly, this is directly attributable to the additional drag flow component.

\subsection{Die-swell flow}

The vector velocity plot of figure 11a shows an initial inlet Poiseuille flow that adjusts to a final plug flow. The radial velocity lines under die-swell conditions (figure $11 \mathrm{~b}$ ), reflect the stick-slip transition at the upper boundary. The contour plots of figures 11b-11e reflect close agreement with the findings of Nickell, et al., ${ }^{11}$ even taking into account the differences in meshing. The radial velocity increases towards the centre of this zone, the maximum value of 0.14 units occurring at the centre. Figure $11 \mathrm{c}$ illustrates contour lines for the axial velocity. It should be noted that $\mathrm{V}_{\mathrm{z}}$ increases at the top boundary after the die exit, whilst on the symmetry axis it diminishes from an inlet value of unity to an exit free jet value of around 0.4 units (figure 12a).

The shear rate $\left(\mathrm{I}_{2}\right)$ line contours of figure $11 \mathrm{e}$ show a localised singularity whose maximum is 10.75 units. In conjunction with figure $12 \mathrm{c}$, we may discern that the shear rate at the top boundary initially commences from a constant value of 1.4 units, but increases exponentially upon nearing the singularity until it peaks at 10.75 units. The shear rate then drops rapidly with further increase in $\mathrm{z}$, departing from the singularity $(z>0)$, to eventually vanish at approximately $z=1.2$ units. The contour plot of pressure in figure $11 \mathrm{~d}$ indicates a maximum inlet value of 4.9 units and minimum value -7.1 units at the singularity. Comparisons are made in table 9, for shear rate maxima $\mathrm{I}_{2}$ and pressure $\mathrm{P}$ extrema, on the three levels of refinement and two different mesh structures. Since the adjustment between coarse and fine mesh results is minor in pressure and minuscule for velocity, plots in the axial direction are shown only for the fine mesh. From table 9, the difference in $\mathrm{I}_{2}$ with mesh refinement is observed to be relatively large in the neighbourhood of the singularity. This is strictly a local phenomenon. On comparing the shear rate profile elsewhere, there is very little difference overall, amounting to one percent at most between the coarse and fine meshes, with no observable difference between the medium and fine versions.

Table 9 Die-swell flow: shear rate and pressure

\begin{tabular}{|cc|c|c|c|c|c|c|}
\hline \multicolumn{2}{|c|}{ Solution } & Cmesh UD & Mmesh UD & Fmesh UD & Cmesh DU & Mmesh DU & Fmesh DU \\
\hline $\mathrm{I}_{2}$ & $\max$ & 5.36 & 8.33 & 10.75 & 6.24 & 9.67 & 12.16 \\
\hline $\mathrm{P}$ & $\min$ & -3.06 & -5.32 & -7.10 & -2.62 & -4.90 & -6.43 \\
& $\max$ & 4.96 & 4.94 & 4.94 & 4.97 & 4.96 & 4.96 \\
\hline
\end{tabular}

On comparing ASSF and die-swell flow for fine meshes in figure $12, \mathrm{I}_{2}$ and pressure profiles are very similar within the die due to the imposition of equivalent inlet 
flow rates for both flows. The shear rate extrema at the singularity achieve maxima of 10.75 units for die-swell flow and 7.93 units for ASSF, representing an increase of thirty six percent (figure 12c). Since for both cases (for the fine UD mesh) stick-slip conditions apply before the die exit, and the flow model lengths are the same, there is little difference between pressure drop values $(0.01 \%)$. On exit from the die the effect of the die-swell on the free surface results in a slight drop in pressure. This is entirely in keeping with our prior results for planar stick-slip flow of figure $7 \mathrm{a}$, where decline rates were contrasted against analytical values. Under the same imposed inlet flow rate, the difference in the velocity on the free surface between axisymmetric stick-slip flow and die swell flow is displayed in figure 12a. The free stream jet exit velocity is twenty one percent lower for die-swell flow than for stick-slip flow. Hence as anticipated, we confirm that allowing the free surface to swell, significantly reduces the flow speed to compensate.

Figure 13a provides the comparison of the derived die-swell surfaces for the six different meshes. The corresponding values for swelling ratio are provided in table 10 , where a direct comparison with results from the literature is performed. Figure 13b shows the effect of mesh refinement on $\mathrm{L}_{\infty}$ error for diagonal orientation meshes DU and UD, for values see table 11 . With mesh refinement, maximum values of $\mathrm{L}_{\infty}$ errors for mesh UD and DU are $\mathrm{O}\left(\mathrm{h}^{1.6}\right)$ and $\mathrm{O}\left(\mathrm{h}^{1.3}\right)$, respectively. The swelling ratio is found to depend on the size of the smallest element and the orientation of the elements. The DU orientation gives approximately fifty percent larger $\mathrm{L}_{\infty}$ error than the UD orientation (see table 11), affecting swelling ratio results accordingly. This superior UD mesh performance is attributed to the richer interpolation offered by the UD orientation for such quantities as velocity gradients, that are represented in a discontinuous distributional sense via the variational treatment (note also the connection to lockingcorner meshing for primary variables). This, we believe pervades many of the solutions reported in the literature, wherever continuous interpolation for primary variables is adopted. Rectangular meshing would suffer from these drawbacks in a likewise manner, being even more restrictive in the variation of functionality offered around the singular point. From table 10, we find that the swelling ratio of the medium refinement meshes, UD and DU, is close to that of Tanner ${ }^{10}$, the error being 0.2 percent for the UD mesh. The swelling ratio of the fine UD mesh is the closest estimate to that of Nickell et al., ${ }^{11}$ with an error of 0.3 percent in that case. Tanner also provides an asymptotic estimate of $\chi=1.130$. This correspondence with the literature may be taken as a strong indication of acceptable accuracy in our results. As indicated above, the orientation of the diagonal element in the mesh that intersects with the singularity, is an important factor and influences the accuracy of the corresponding solutions. To demonstrate this issue the error in the swelling ratio is charted in table 11 against Nickell et al. results, on the two DU and UD mesh sets. Trends in convergence are clearly superior for the UD mesh sets in comparison to those for DU. A hybrid fine mesh strategy also implemented, of DU in the die (Figure 10f) and UD in the jet (Figure $10 \mathrm{c}$ ), gave a marginal improvement over the UD option in swelling ratio to reach the asymptotic value of $\chi=1.30$. It is also noted that the separation angle, $\theta$, between the horizontal and the exterior swelling edge of the first element after the singular point, is smaller for the UD meshes. This angle tends to a value of $17.5^{\circ}$ with mesh refinement on the UD meshes, as compared to $20.8^{\circ}$ for the DU alternative. 
Table 10 Swelling ratio for die-swell flow

\begin{tabular}{|c|c|c|}
\hline Investigator & $\chi$ & $\chi(\%)$ \\
\hline Tanner $^{10}$ & 1.136 & 13.6 \\
\hline Nickell et al. $^{11}$ & 1.128 & 12.8 \\
\hline Chang et al. $^{12}$ & 1.139 & 13.9 \\
\hline Crochet and Keunings $^{13}$ & 1.126 & 12.6 \\
\hline Coarse UD mesh $^{\text {Medium UD mesh }}$ & 1.141 & 14.1 \\
\hline Fine UD mesh & 1.134 & 13.4 \\
\hline Coarse DU mesh & 1.131 & 13.1 \\
\hline Medium DU mesh & 1.147 & 14.7 \\
\hline Fine DU mesh & 1.137 & 13.7 \\
\hline Fine DU_UD mesh & 1.133 & 13.3 \\
\hline
\end{tabular}

Table 11 Swelling ratio error for UD and DU meshes against Nickell et al. ${ }^{11}$ results

\begin{tabular}{|l|c|c|c|}
\hline \multicolumn{1}{|c|}{ Mesh } & $\mathrm{L}_{\infty}$ error*10-2 & $\theta$ (degree) & $\Delta \mathrm{h}^{*} 10^{-2}$ \\
\hline (a) Coarse UD mesh & 1.1525 & 18.2881 & 6.4550 \\
\hline (b) Medium UD mesh & 0.5319 & 17.8016 & 3.0533 \\
\hline (c) Fine UD mesh & 0.2660 & 17.5737 & 1.9667 \\
\hline (d) Coarse DU mesh & 1.6944 & 21.4798 & 6.4550 \\
\hline (e) Medium DU mesh & 0.7979 & 20.9369 & 3.0533 \\
\hline (f) Fine DU mesh & 0.4433 & 20.4761 & 1.9667 \\
\hline Fine DU_UD mesh & 0.1525 & 17.6604 & 1.9667 \\
\hline
\end{tabular}

In contrast, on testing the Phan-Thien free surface procedure, correspondingly larger swelling ratios are derived. It has been found necessary to impose an additional velocity free surface boundary correction with this procedure to ensure tangential conditions and vanishing shear stress. Without such a correction, the results on swell and angle are considerably inaccurate. Table 12 displays swelling ratio and angle computed on coarse and fine UD meshes. The corresponding swelling ratios are 35.4 and 35.0 percent, respectively, with separation angles of $23.7^{\circ}$ for the coarse and $23.6^{\circ}$ for the fine mesh. Both of these estimates are marked in their departure from the results of Nickell et al. and other investigators. For example, on the fine mesh, the error in swelling ratio from that of Nickell et al. is 19.7 percent. Hence for current purposes, and as implemented here in a pointwise fashion following the original author, this method is discarded on the grounds of inaccuracy.

Table 12 Swelling ratio and angle for Phan-Thien strategy

\begin{tabular}{|c|c|c|}
\hline Mesh & $\chi$ & $\theta$ (degree) \\
\hline Coarse UD mesh & 1.354 & 23.6660 \\
\hline Fine UD mesh & 1.350 & 23.6121 \\
\hline
\end{tabular}




\subsection{Die-swell/drag flow}

For this final flow instance under consideration, we allot for mesh (c) of table 2 and figure 10c, as the swelling ratio errors on UD meshes are considerably lower than those for the DU meshes. A principal point of interest is to analyse the effect of the additional component of drag flow upon the undisturbed die-swell flow. In this regard, comparison is made in table 13 for shear rate $\mathrm{I}_{2}$ maxima and observed extrema for pressure $\mathrm{P}$. For die-swell/drag flow, shear rate $\mathrm{I}_{2}$ and pressure drop are reduced when compared with die-swell flow by 21 and 19.6 percent, respectively. The contours of figure 14 bear this out. For figure $14 \mathrm{a}$ in contrast to figure 11d, the pressure contours reflect the reduced effect on the negative pressure pockets (-5.58 units) near the singularity over the die-swell case (-7.1 units). Likewise, values of shear rate maxima alter from figure $14 \mathrm{~b}$ (8.47 units) to that for die-swell in figure 11e (10.75 units).

The finer and more localised detail comparing these two flow scenarios is extracted in the line plots of figure 15. Figure $15 \mathrm{c}$ illustrates the pressure $\mathrm{P}$ on the inner surface, and figure $15 \mathrm{~d}$ the shear rate $\left(\mathrm{I}_{2}\right)$ at the free surface. The line pressure plot reveals that drag flow gives rise to a negative dip in pressure on the inner surface beyond the die exit. This was not present when drag flow was introduced for stick-slip flows (see figure 9b), and so is a consequence of the die-swell setting. Also, the decline in pressure drop is prominent. The shear rate profile of figure $15 \mathrm{~d}$ (table 13) can be compared against both figure 9c, for ASSF-SSDF (table 8), and figure 12c, for ASSF-die-swell. In the die-swell setting, drag flow incurs a reduction in peak $\mathrm{I}_{2}$ values (10.75 to 8.47 units), comparable to the effect noted for stick-slip (7.93 to 6.28 units). Alternatively, in the contrast between die-swell and stick-slip (with or without drag flow) there is a consistent trend in elevation of peak $I_{2}$ values once swelling is present (here by thirty five percent).

Table 13 Die-swell flow and die-swell/drag flow: shear rate and pressure

\begin{tabular}{|ccc|c|c|}
\hline \multicolumn{2}{|c|}{ Solution } & die-swell flow & die-swell/drag flow \\
\hline $\mathrm{I}_{2}$ & $\max$ & 10.75 & 8.47 \\
\hline $\mathrm{P}$ & $\min$ & -7.10 & -5.58 \\
& $\max$ & 4.94 & 3.97 \\
\hline
\end{tabular}

The radial and axial velocity contour plot for the die-swell/drag flow are similar in appearance to figures $11 \mathrm{~b}$ and $11 \mathrm{c}$, and are not reproduced for the sake of conciseness. Figure $15 \mathrm{a}$ and $15 \mathrm{~b}$ provide line plot comparisons between die-swell flow and die-swell/drag flow for velocity and die swell, respectively, at the free surface employing the fine UD mesh. At the outlet, the free stream velocity of die-swell/drag flow is reduced by twenty one percent over that of pure die-swell, so that swelling reduces accordingly by 4.2 percent. This is in keeping with the correspondence in flow rate at the outlet and our findings for the stick-slip scenario. The separation angle for die-swell/drag flow is $17.19^{\circ}$, which is a reduction of 2.2 percent on the former dieswell case. This accounts for the above quoted reduction in swelling ratios between these two flow instances.

\section{CONCLUSIONS}

This study has provided an analysis of a Taylor-Galerkin/Pressure-Correction method in its application for model free surface flow problems. First, through the investigation of stick-slip flow we have been able to establish comparison against analytical and other numerical solutions, for which we find agreement to within order one percent. 
For die-swell flows, with the added complication of apriori unknown free surface location, we find close correspondence on swelling ratio to that reported in the literature, to within order 0.1 percent. Through a careful study of mesh structure, we have also found that the accuracy of the solutions generated is sensitive to the orientation of the mesh in the location of the die exit. Here, we have demonstrated that a poor selection of meshing may affect accuracy by up to fifty percent. Accuracy has been demonstrated to pertain to second-order, with or without free surface location involved. This is so even in the presence of a die-exit singularity to the flow in question.

In the comparison of stick-slip to die-swell flows under equivalent imposed inlet flow rate, the free stream jet velocity is twenty one percent lower for die-swell flow than for stick-slip flow, whilst pressure profiles barely differ. The shear rate extrema at the singularity peak at 10.75 units for die-swell flow, but attain the lower value of 7.93 units for stick-slip flow. Hence, shear rate extrema are elevated by thirty five percent once die-swell is incorporated.

We have also addressed the issue of associating an additional drag flow component to these two base type flows. This has afforded the opportunity to compare scenarios both with and without drag flow. Our findings reveal that shear rates at the singularity are reduced by as much as twenty one percent with the addition of drag flow for both slick-slip and die-swell flows. It is conspicuous that the same level of reduction in shear rate is observed for both flows. This we attribute to the local influence at the singularity that the inclusion of drag flow has. Likewise, pressure drops are also found to decrease by 17.6 percent for stick-slip and 19.6 percent for dieswell flow. In the die-swell instance alone, the swelling ratio is observed to reduce by 4.2 percent upon the addition of drag flow.

This research study may be viewed as a stepping stone towards the solution of more complex industrial based flows that involve coatings of one form or another. This is typically the case for example in processes such as wire coating, roller-coating and printing. 


\section{APPENDIX}

To derive the annular inlet flow profile, we follow $\operatorname{Bird}^{23}$ and use the nondimensional equation for annular pressure driven flow,

$$
\mathrm{V}_{\mathrm{z}}(\mathrm{r})=\frac{\mathrm{Pb}^{2}}{4 \mu \mathrm{L}}\left\{1-\left(\frac{\mathrm{r}}{\mathrm{b}}\right)^{2}+\frac{1}{\ln \left(\frac{\mathrm{a}}{\mathrm{b}}\right)}\left[\left(\frac{\mathrm{a}}{\mathrm{b}}\right)^{2}-1+\frac{4 \mu \mathrm{LV} \text { wire }}{\mathrm{Pb}^{2}}\right] \ln \left(\frac{\mathrm{r}}{\mathrm{b}}\right)\right\}
$$

where variables are defined as viscosity $\mu$, length $Z_{1} Z_{2}$ (figure $1 b$ ) $\mathbf{L}$, pressure drop between inlet and outlet $\mathbf{P}$, wire speed $\mathbf{V}_{\text {wire }}$, inner annular radius $\mathbf{a}$ and outer radius $\mathbf{b}$.

Subsequently, we may derive the flowrate at inlet and relate this to pressure drop, via

$$
\mathrm{Q}=2 \pi \int_{\mathrm{a}}^{\mathrm{b}} \mathrm{rV}_{\mathrm{z}}(\mathrm{r}) \mathrm{dr} \text {. }
$$

Hence, once flowrate Q is prescribed (say from an outlet plug or free jet flow), we may evaluate the pressure drop from the constant term, $\frac{\mathrm{Pb}^{2}}{4 \mu \mathrm{L}}$, utilising equation (A.1) for $\mathrm{V}_{\mathrm{z}}(\mathrm{r})$ within equation (A.2).

We have recourse to the stream function solution for planar stick-slip flow, as developed in the article of Richardson ${ }^{3}$ :

\section{$\underline{\text { case } x>0}$}

$\psi(x, y)=y-\frac{3}{2 \pi} \sum_{n=1}^{\infty} \frac{(-1)^{n}}{B_{-}(-i n \pi)} x e^{-n \pi x} \sin n \pi y+\frac{3}{2} \sum_{n=1}^{\infty}(-1)^{n} \underset{w \rightarrow-i n \pi}{\operatorname{Lt}}\left[\frac{d}{d w}\left\{\frac{i}{w^{2} B_{-}(w)}\right\}\right] e^{-n \pi x} \sin n \pi y$

$\underline{\text { case } x<0}$

$$
\psi(x, y)=\frac{1}{2} y\left(3-y^{2}\right) y-3 \sum_{n=1}^{\infty} \Re\left\{\frac{B_{+}\left(\frac{1}{2} \alpha_{n}\right)}{\sinh ^{2}\left(\frac{1}{2} \alpha_{n}\right)}\left[\frac{\sinh \left(\frac{1}{2} \alpha_{n} y\right)}{\sinh \left(\frac{1}{2} \alpha_{n}\right)}-\frac{y \cos \left(\frac{1}{2} \alpha_{n} y\right)}{\cosh \left(\frac{1}{2} \alpha_{n}\right)}\right] e^{-i \frac{1}{2} \alpha_{n} x}\right\}
$$

where $B_{+}(w)=-\frac{1}{3} \prod_{n=1}^{\infty} \frac{\left(1+\frac{2 w}{\alpha_{n}}\right)\left(1-\frac{2 w}{\overline{\alpha_{n}}}\right)}{\left(1+\frac{\mathrm{w}}{\mathrm{in} \pi}\right)^{2}} \quad$ and $\quad B_{-}(w)=\prod_{n=1}^{\infty} \frac{\left(1-\frac{2 \mathrm{w}}{\alpha_{\mathrm{n}}}\right)\left(1+\frac{2 \mathrm{w}}{\alpha_{\mathrm{n}}}\right)}{\left(1-\frac{\mathrm{w}}{\mathrm{in} \pi}\right)^{2}}$,

$$
\alpha_{\mathrm{n}} \approx \ln 2 \beta_{\mathrm{n}}+\left(\beta_{\mathrm{n}}-\frac{\ln 2 \beta_{\mathrm{n}}}{\beta_{\mathrm{n}}}\right) \mathrm{i} \quad \text { and } \quad \beta_{\mathrm{n}}=2 \mathrm{n} \pi+\frac{1}{2} \pi, \quad \mathrm{n}=1,2,3, \ldots
$$

To analyse accuracy in terms of mesh size, we may express the finite element solution for velocity as a power series expansion about the analytical solution,

$$
\mathrm{U}^{\mathrm{fe}}(\Delta \mathrm{h})=\mathrm{U}^{\mathrm{anal}}+\mathrm{C}(\Delta \mathrm{h})^{\alpha} .
$$

This allows us to consider a $\mathrm{L}_{\infty}$ relative error measure for velocity against the analytical solution of Richardson on various meshes, each denoted by element size $\Delta \mathrm{h}$,

$$
\frac{\max \left|\mathrm{U}^{\mathrm{Anal}}-\mathrm{U}^{\mathrm{Fe}}\right|}{\max \left|\mathrm{U}^{\text {Anal }}\right|}
$$

Here, variables are defined as the numerical solution $\mathrm{U}^{\mathrm{Fe}}$, the analytical solution $\mathrm{U}^{\text {Anal }}$, size of the smallest element $\Delta h$, constant $C$, and the order of error constant $\alpha$. 


\section{REFERENCES}

1. A. Baloch, P. Townsend and M. F. Webster, 'On Two- and Three-Dimensional Expansion Flows', Comp. \& Fluids, vol 24 no. 8, pp 863-882 (1995).

2. P. Rameshwaran, P. Townsend and M. F. Webster, 'Simulation of Particle Setting in Rotating and Non-Rotating Flow of Non-Newtonian Fluids', Int. J. Num. Meth. Fluids, vol 26, pp 851-874 (1998).

3. S. Richardson, "A "Stick-Slip" Problem Relatived to the Motion of a Free Jet at Low Reynolds Numbers', Proc. Camb. Phil. Soc., vol 67, pp 477-489 (1970).

4. M. Okabe, 'Fundamental Theory of the Semi-Radial Singularity Mapping with Applications to Fracture Mechanics', Comp. Meth. App. Mech. Eng., vol 26, pp 53-73 (1981).

5. D. B. Ingham and M.A. Kelmanson, 'Boundary Integral Equation Analyses of Singular Potential and Biharmonic Problems', Spinger-Verlag Berlin, pp 21-51 (1984).

6. M. Kermode, A. Mckerrell and L. M. Delves, 'The Calculation of Singular Coefficients', Comp. Meth. App. Mech. Eng., vol 50, pp 205-215 (1985).

7. C. Cuvelier, A. Segal and A. A. van Steenhoven, 'Finite Element Methods and Navier-Stokes Equations', D. Reidel Publishing Company, 1986.

8. G. C. Georgiou, L. G. Olson, W. W. Schultz and S. Sagan, 'A Singular Finite Element for Stokes Flow: The Stick-Slip Problem', Int. J. Num. Meth. Fluid, vol 9, pp 1353-1367 (1989).

9. G. Georgiou, L. Olson and W. Schultz, 'The Integrated Singular Basis Function Method for the Stick-Slip and the Die-Swell Problem', Int. J. Num. Meth. Fluids, vol 13, pp 1251-1265 (1991).

10. R. I. Tanner, 'Engineering Rheology', Oxford University Press, London, 1985.

11. R. E. Nickell, R. I. Tanner and B. Caswell, 'The Solution of Viscous Incompressible Jet and Free Surface Flows Using Finite-Element Methods', J. Fluid Mech., vol 65, part 1, pp 189-206 (1974).

12. P. W. Chang, T. W. Patten and Finlayson, 'Collocation and Galerkin Finite Element Methods for Viscoelastic fluid Flow-II', Comp. and Fluids, vol 17, pp 285-293 (1979).

13. M. J. Crochet and R. Keunings, 'Die Swell of a Maxwell Fluid Numerical Prediction', J. Non-Newtonian Fluid Mech., vol 7, pp 199-212 (1980).

14. M. J. Crochet and R. Keunings, 'On Numerical Die Swell Calculation', J. nonNewtonian Fluid Mech., vol 10, pp 85-94 (1982).

15. W. J. Silliman and L. E. Scriven, 'Separating Flow Near a Static Contact Line: Slip at a Wall and Shape of a Free Surface', J. Comp. Phys., vol 34, pp287-313 (1980).

16. V. Ngamaramvaranggul and M. F. Webster, 'Simulation of Coating Flows with Slip Effects', under submission to Int. J. Num. Meth. Fluids, (1999).

17. N. Phan-Thien, 'Influence of Wall Slip on Extrudate Swell: a Boundary Element Investigation', J. Non-Newtonian Fluid Mech., vol 26, pp 327-340 (1988).

18. C. R. Beverly and R. I. Tanner, 'Numerical Analysis of Three-Dimensional Newtonian extrudate Swell', Rheol. Acta, vol 30, pp 341-356 (1991).

19. A. Karagiannis, A. N. Hrymak and J. Vlachopoulos, 'Three-dimensional NonIsothermal Extrusion Flows', Rheol. Acta, vol 28, pp 121-133 (1989).

20. C. W. Butler and M. B. Bush, 'Extrudate Swell in Some Dilute Elastic Solution', Rheol. Acta, vol 28, pp 294-301 (1989).

21. R. Ahmed, R. F. Liang and M. R. Mackley, 'The Experimental Observation and Numerical Prediction of Planar Entry Flow and Die Swell for Molten Polyethylenes', J. Non-Newtonian Fluid Mech., vol 59, pp 129-153 (1995).

22. M. J. Crochet, A. R. Davies, K. Walters, 'Numerical Simulation of NonNewtonian Flow', Rheology Series 1, Elsevier Science Publishers, 1984.

23. R. B. Bird, W. E. Steward and E. N. Lightfoot, 'Transport Phenomena', John Wiley \& Sons, 1960. 


\section{FIGURE LEGEND}

Table 1 Finite element meshes for stick-slip flow

Table 2 Finite element meshes for die-swell flow

Table 3 Shear rate and pressure for planar stick-slip flow: various meshes

Table 4 Analytical and computed velocity along axis of symmetry: various meshes

Table 5 Analytical and computed pressure along axis of symmetry: various meshes

Table $6 \mathrm{~L}_{\infty}$ error for velocity and pressure against analytical solution: various meshes

Table 7 Analytical and computed velocity results at $x=0.2$ on free surface after die exit

Table 8 Axisymmetric stick-slip and stick-slip/drag flow: shear rate and pressure

Table 9 Die-swell flow: shear rate and pressure

Table 10 Swelling ratio for die-swell flow

Table 11 Swelling ratio error for UD and DU meshes against Nickell et al. ${ }^{11}$ results

Table 12 Swelling ratio and angle of Phan-Thien strategy

Table 13 Die-swell flow and die-swell/drag flow: shear rate and pressure 
FIGURE LEGEND (continued)

Figure 1: Schema for flow problems

(a) stick-slip, (b) stick-slip/drag, (c) die-swell

Figure 2: Planar stick-slip flow: mesh patterns

(a) coarse mesh, 5x20 elements, (b) medium mesh, 10x40 elements,

(c) fine mesh, 15x60 elements, (d) biased fine mesh, 15x60 elements

Figure 3: Velocity results for planar stick-slip flow: medium mesh, $\mathrm{Re}=0$

(a) velocity vectors, (b) $\mathrm{V}_{\mathrm{x}}$ contours, (c) $\mathrm{V}_{\mathrm{y}}$ contours

Figure 4: Planar stick-slip flow: medium mesh, $\mathrm{Re}=0$

(a) pressure contours, (b) $I_{2}$ contours, (c) $I_{2}$ on free surface

Figure 5: Planar stick-slip flow: medium mesh, cross-channel velocity profiles, $R e=0$

(a) $\mathrm{x} \leq 0$, (b) $\mathrm{x} \geq 0$

Figure 6: Planar stick-slip flow: analytical and numerical solutions for velocity field along centreline $y=0, \operatorname{Re}=0$

(a) $x<0$, (b) $x>0$

Figure 7: Planar stick-slip flow: analytical and numerical solutions, $\mathrm{Re}=0$

(a) pressure line plot along centreline $\mathrm{y}=0$,

(b) velocity at free surface $(0<x<0.3)$,

(c) velocity error norms against Richardson [3]

Figure 8: Stick-slip/drag flow: $\operatorname{Re}=10^{-4}$

(a) mesh pattern, 18x54 elements, (b) velocity vectors,

(c) pressure contours, (d) $\mathrm{I}_{2}$ contours

Figure 9: Stick-slip flow and stick-slip/drag flow, $\mathrm{Re}=10^{-4}$

(a) $\mathrm{V}_{\mathrm{z}}$ on free surface,

(b) pressure on axis of symmetry for stick-slip flow and inner surface for stick-slip/drag flow, (c) $I_{2}$ on free surface

Figure 10: Die-swell flow: mesh patterns

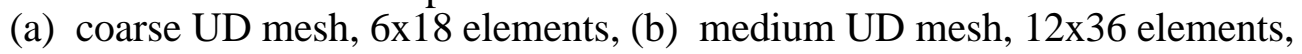

(c) fine UD mesh, 18x54 elements, (d) coarse DU mesh, 6x18 elements,

(e) medium DU mesh, $12 \times 36$ elements, (f) fine DU mesh, 18x54 elements

Figure 11: Die-swell flow: fine UD mesh, $\mathrm{Re}=10^{-4}$

(a) velocity vectors, (b) $\mathrm{V}_{\mathrm{r}}$ contours, (c) $\mathrm{V}_{\mathrm{z}}$ contours,

(d) pressure contours, (e) $\mathrm{I}_{2}$ contours

Figure 12: Stick-slip flow and die-swell flow, fine UD mesh, $\operatorname{Re}=10^{-4}$

(a) $\mathrm{V}_{\mathrm{z}}$ on free surface,

(b) pressure on axis of symmetry for stick-slip flow and die-swell flow,

(c) $\mathrm{I}_{2}$ on free surface

Figure 13: Die-swell flow: comparison of solutions, $\operatorname{Re}=10^{-4}$

(a) swell free surface with mesh refinement,

(b) die-swell error norms against Nickell et al. [11]

Figure 14: Die-swell/drag flow: fine UD mesh, $\mathrm{Re}=10^{-4}$

(a) pressure contours, (b) $I_{2}$ contours

Figure 15: Die-swell flow and die-swell/drag flow, fine UD mesh

(a) velocity, (b) die swell,

(c) pressure on axis of symmetry for die-swell flow and inner surface for die-swell/drag flow, (d) $I_{2}$ on free surface 


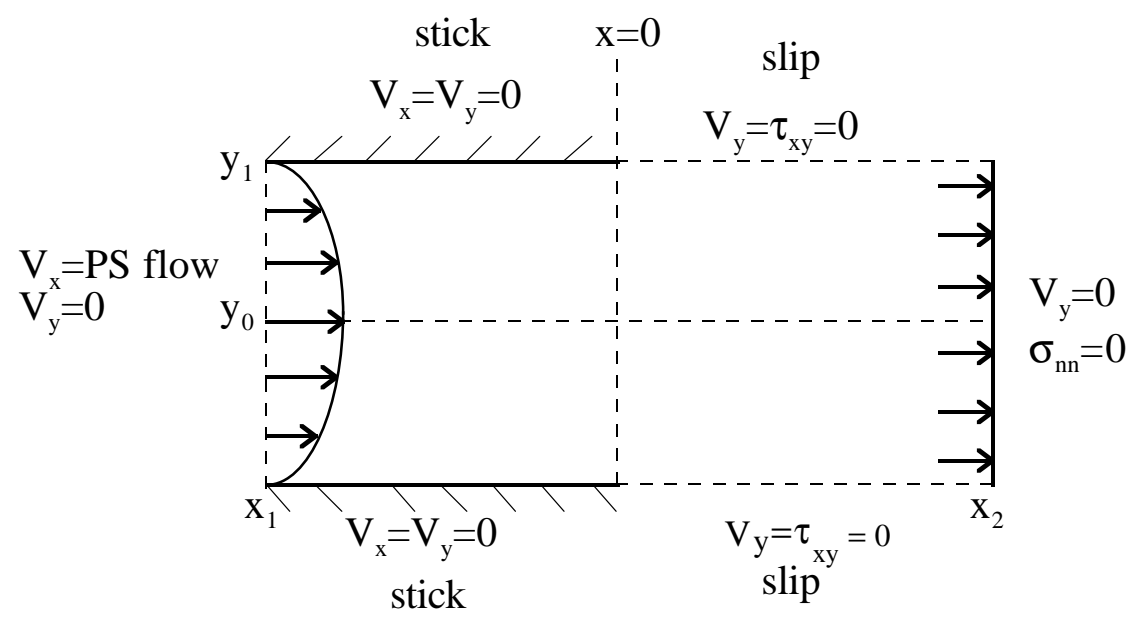

(a) stick-slip

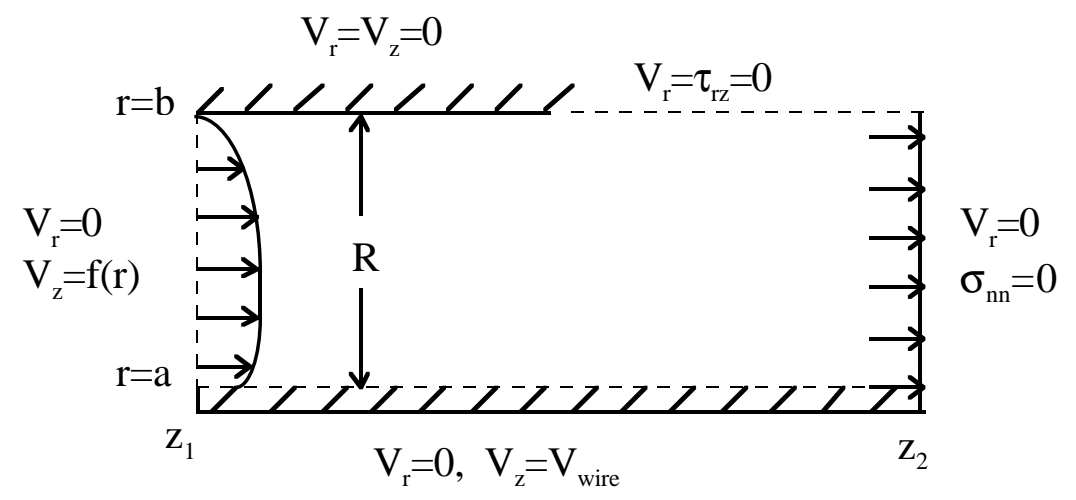

(b) stick-slip/drag

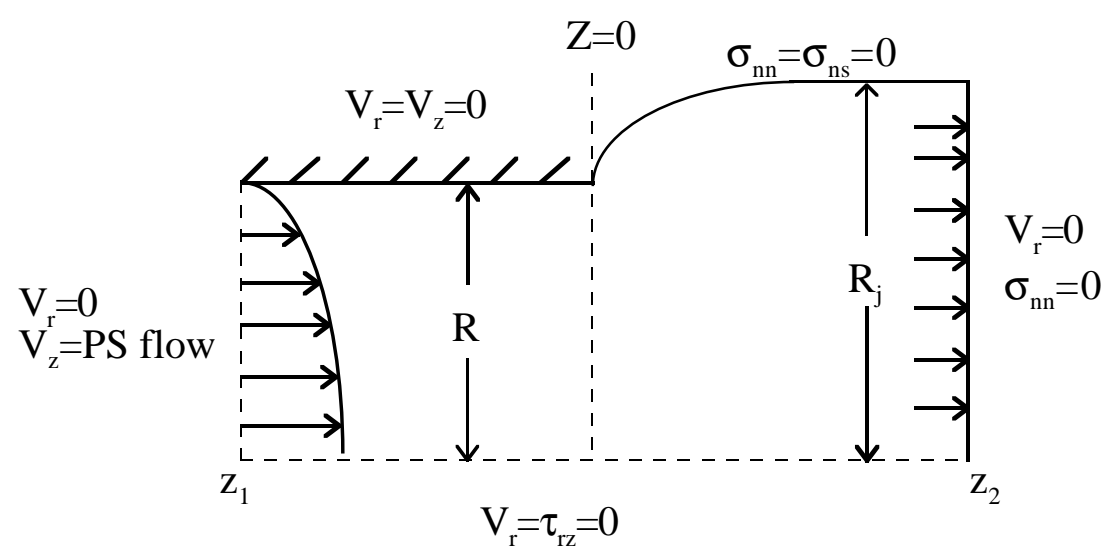

(c) die-swell

Figure 1: Schema for flow problems 


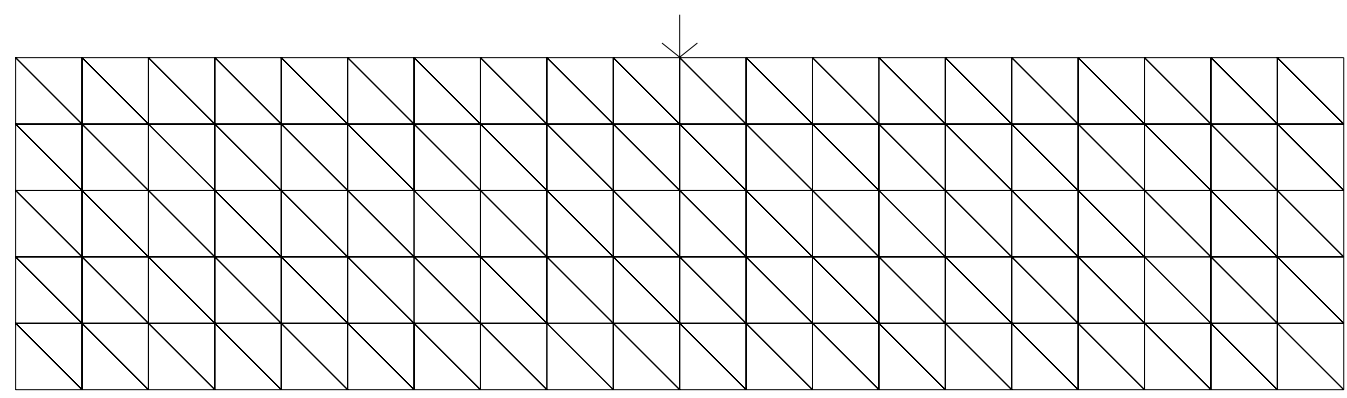

(a) coarse mesh, 5x20 elements

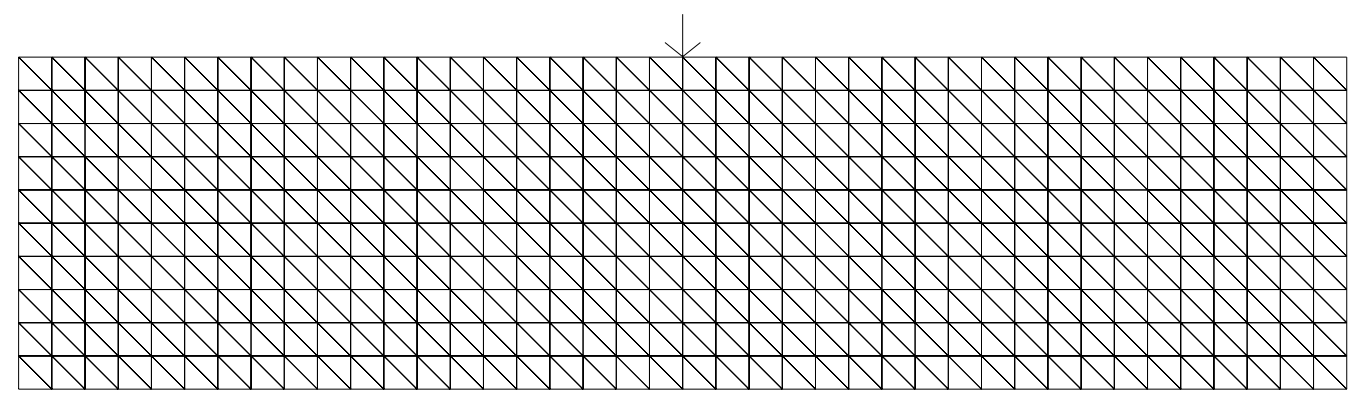

(b) medium mesh, 10x40 elements

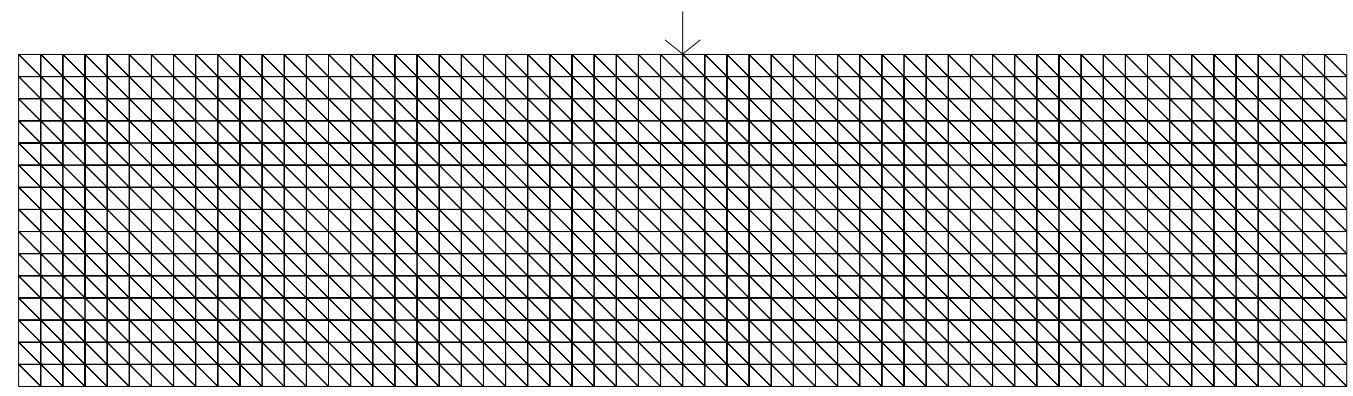

(c) fine mesh, 15x60 elements

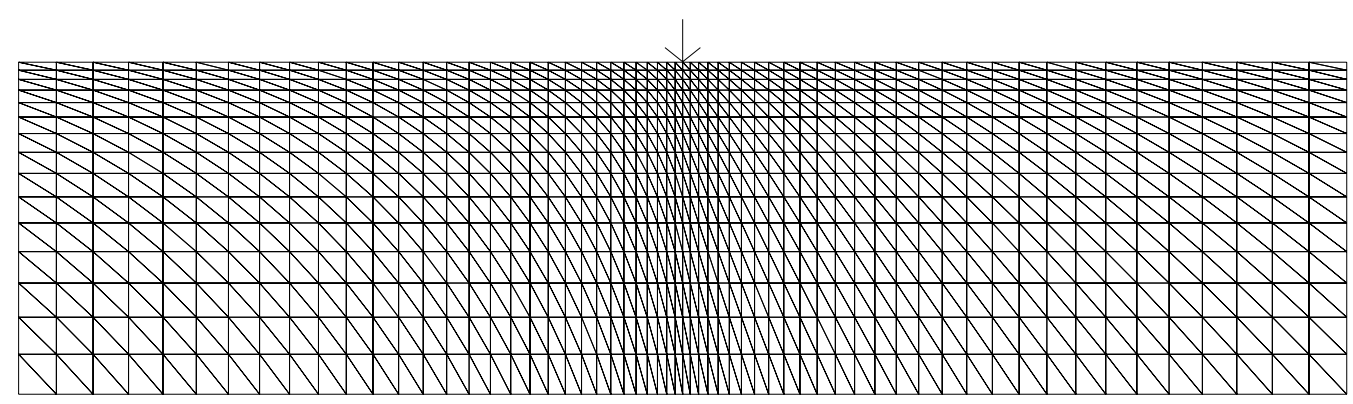

(d) biased fine mesh, 15x60 elements

Figure 2: Planar stick-slip flow: mesh patterns 


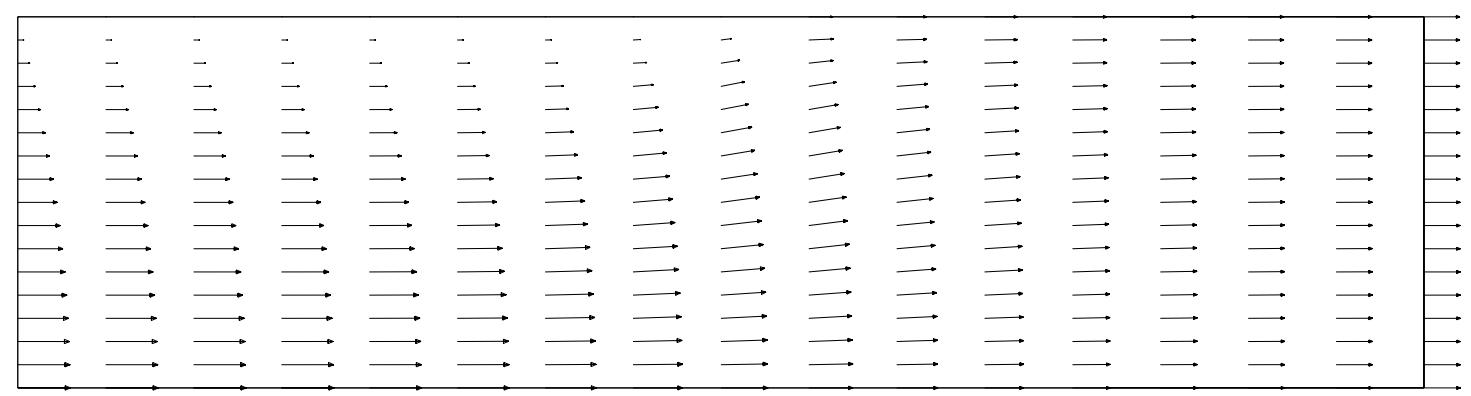

(a) velocity vectors
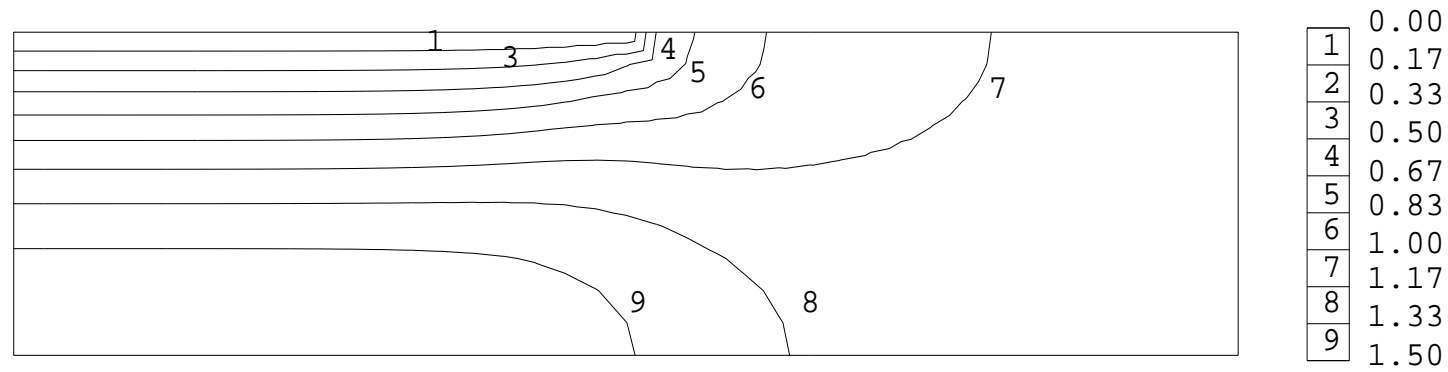

(b) $V_{x}$ contours
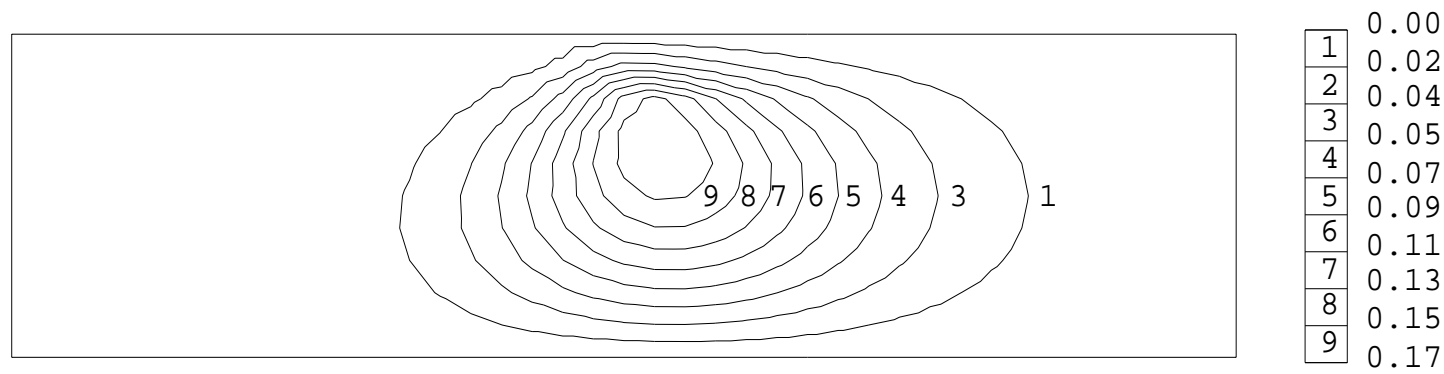

(c) $V_{y}$ contours

Figure 3: Velocity results for planar stick-slip flow: medium mesh, $R e=0$ 


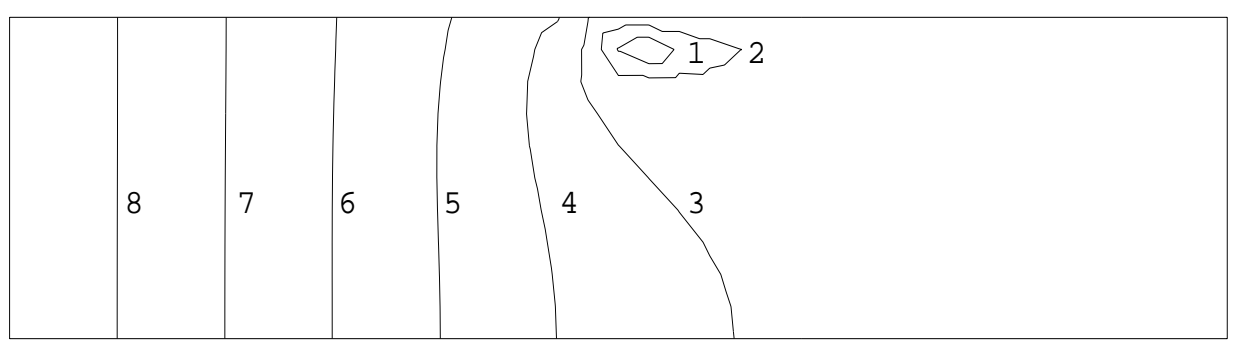

$-2.8$

$-1.7$

$-0.6$

0.4

\begin{tabular}{l|l}
4 & 1.5
\end{tabular}

52.6

6.7

7.7

8 ( 5.8

96.9

(a) pressure contours

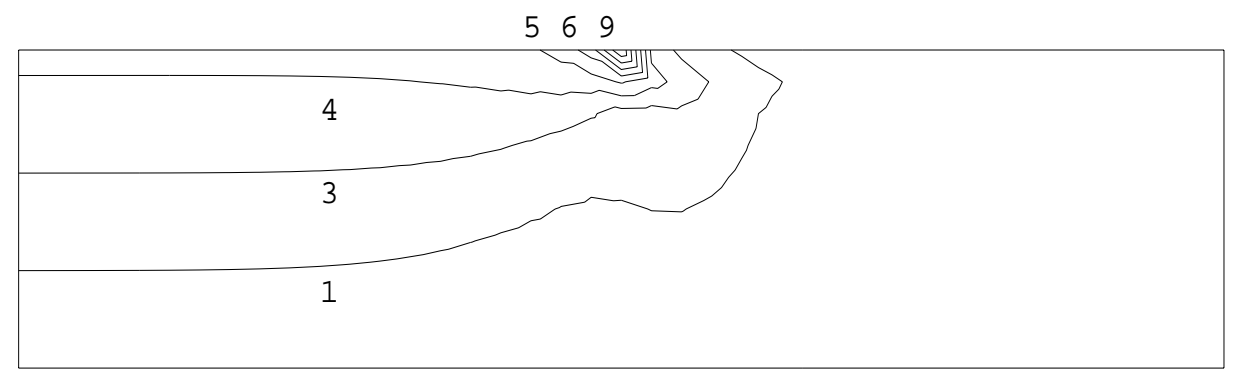

\begin{tabular}{|c|}
\hline 1 \\
\hline 2 \\
\hline 3 \\
\hline 4 \\
\hline 5 \\
\hline 6 \\
\hline 8 \\
\hline 9 \\
\hline
\end{tabular}

(b) $I_{2}$ contours

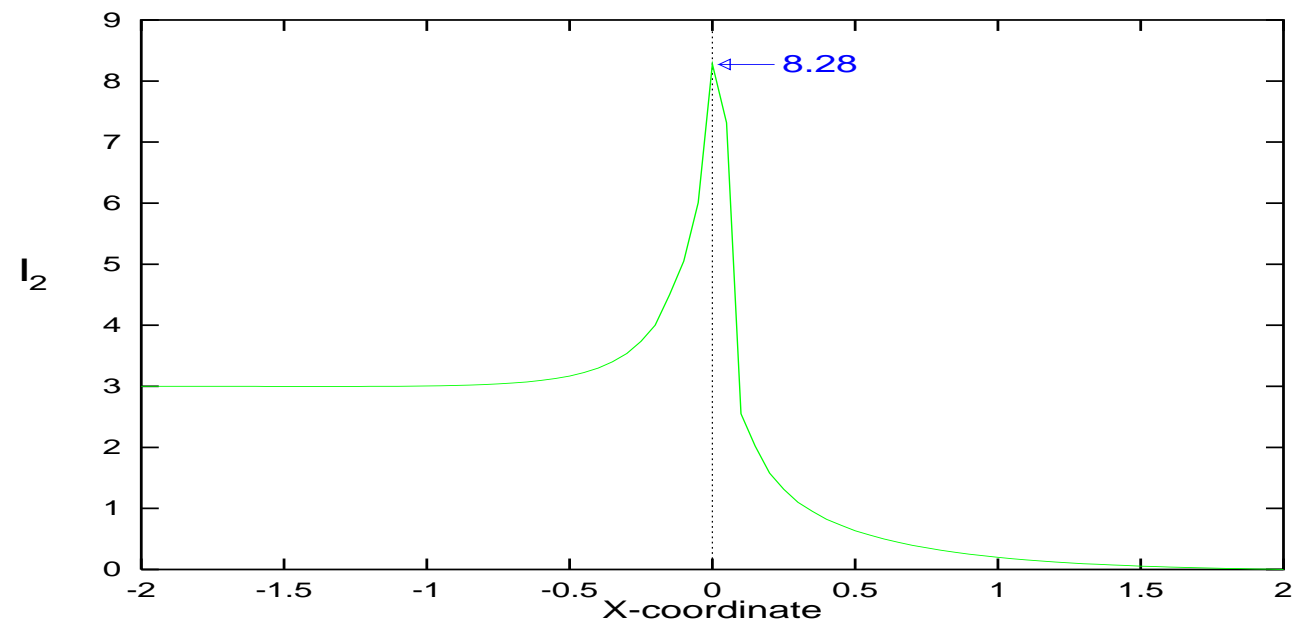

(c) $I_{2}$ on free surface

Figure 4: Planar stick-slip flow: medium mesh, $\mathbf{R e}=\mathbf{0}$ 


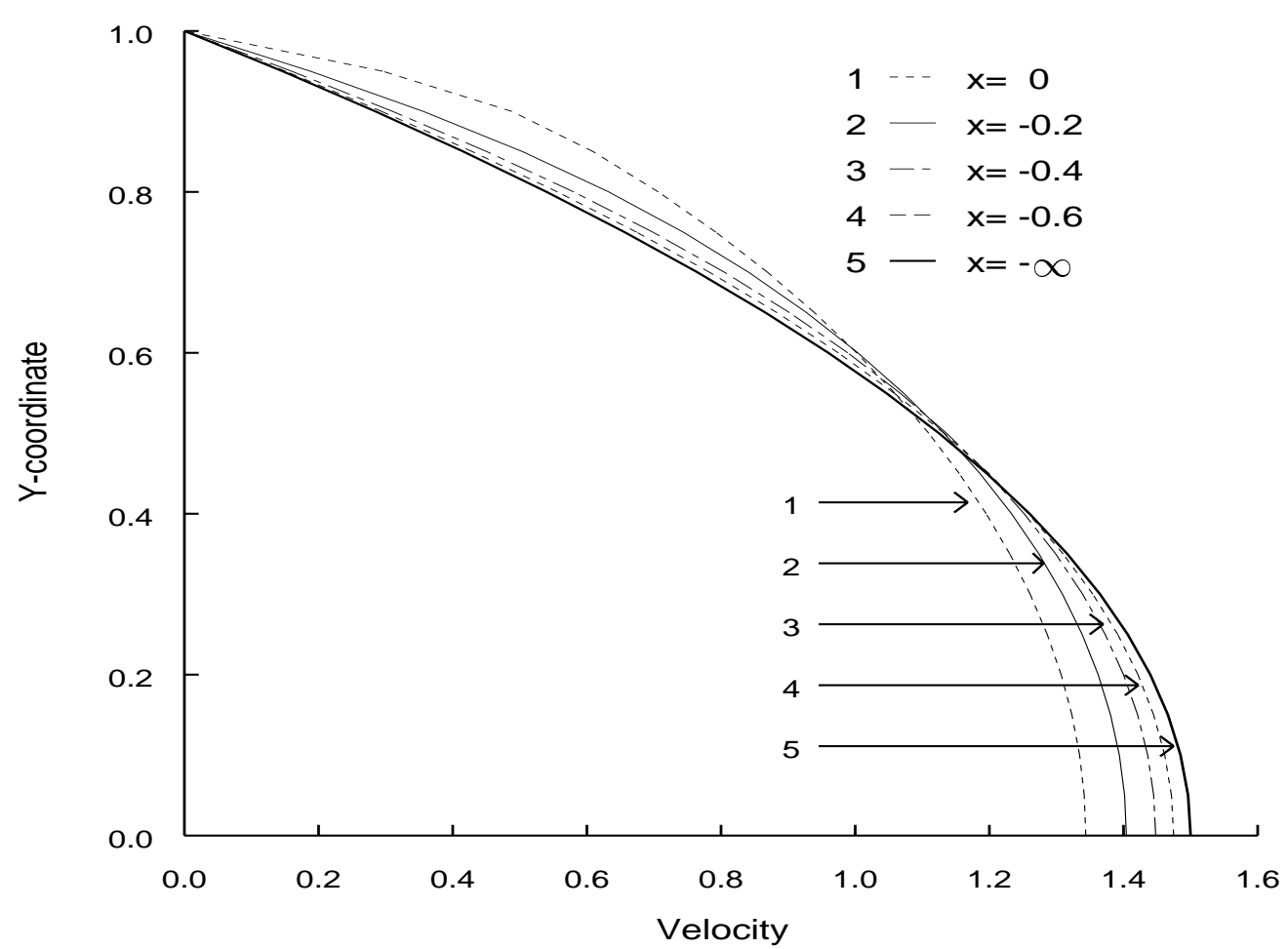

(a) $\mathbf{x} \leq \mathbf{0}$

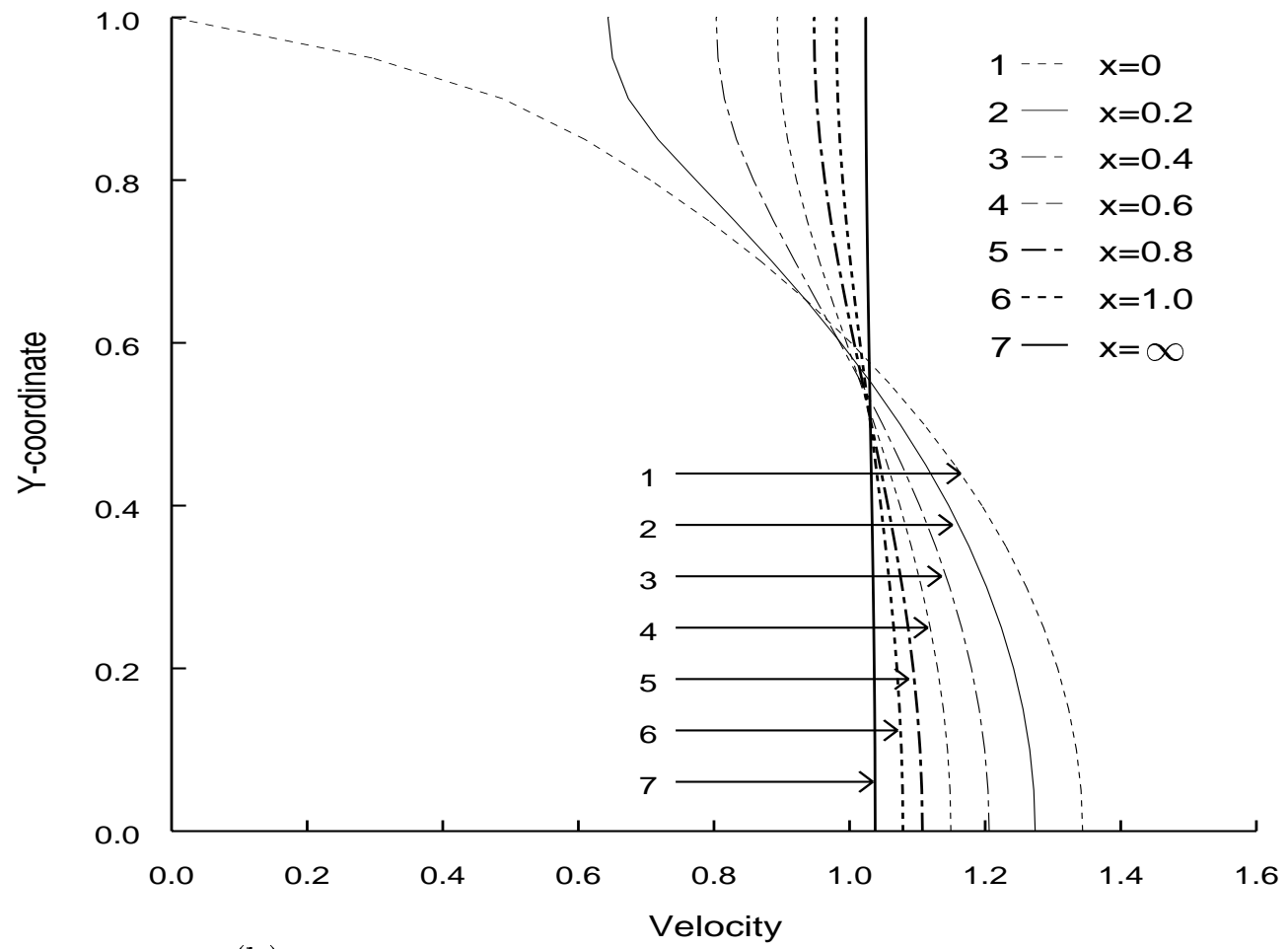

(b) $\mathbf{x} \geq \mathbf{0}$

Figure 5: Planar stick-slip flow: medium mesh, cross-channel velocity profiles, $\mathrm{Re}=\mathbf{0}$ 


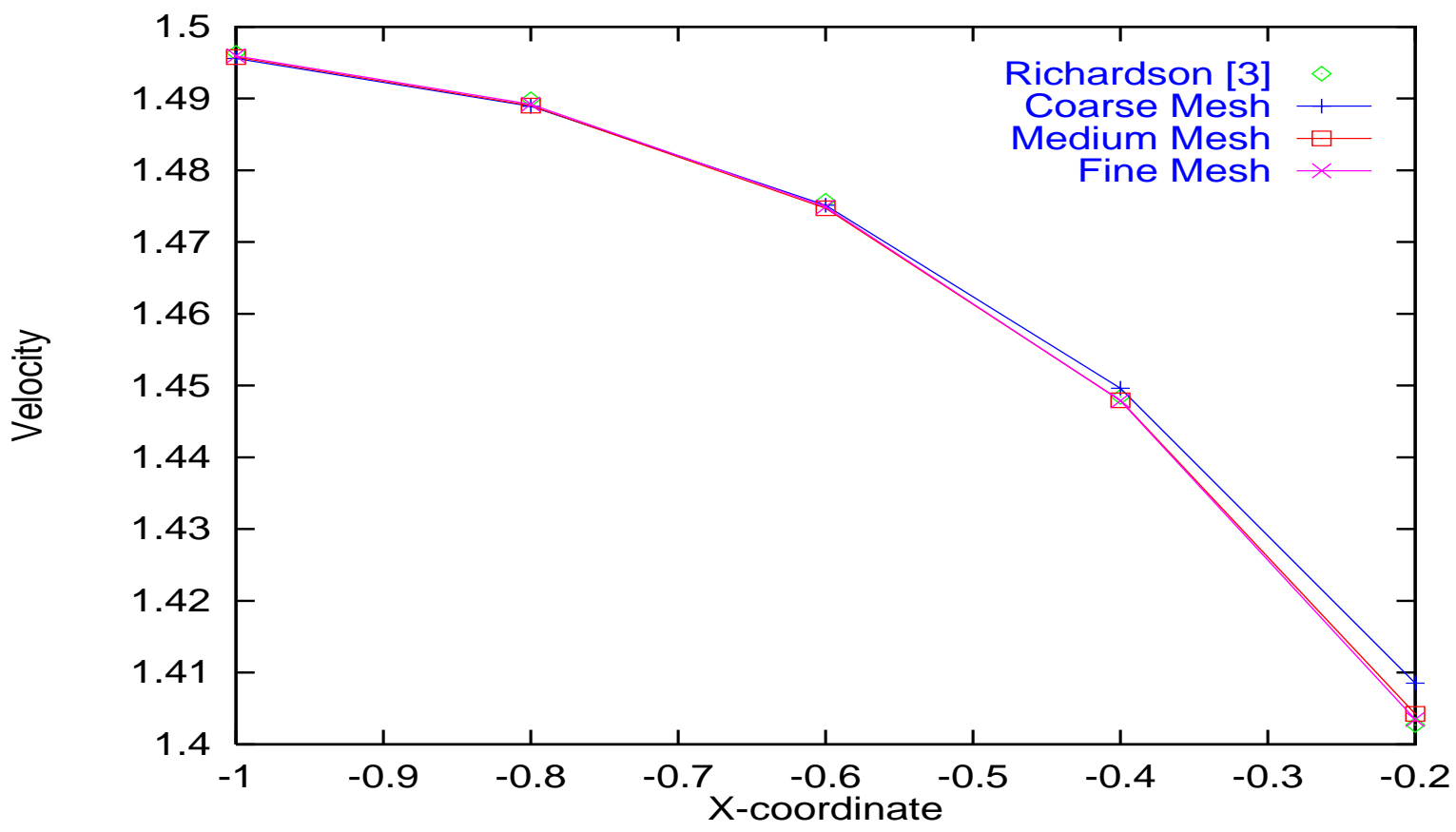

(a) $\mathbf{x}<\mathbf{0}$

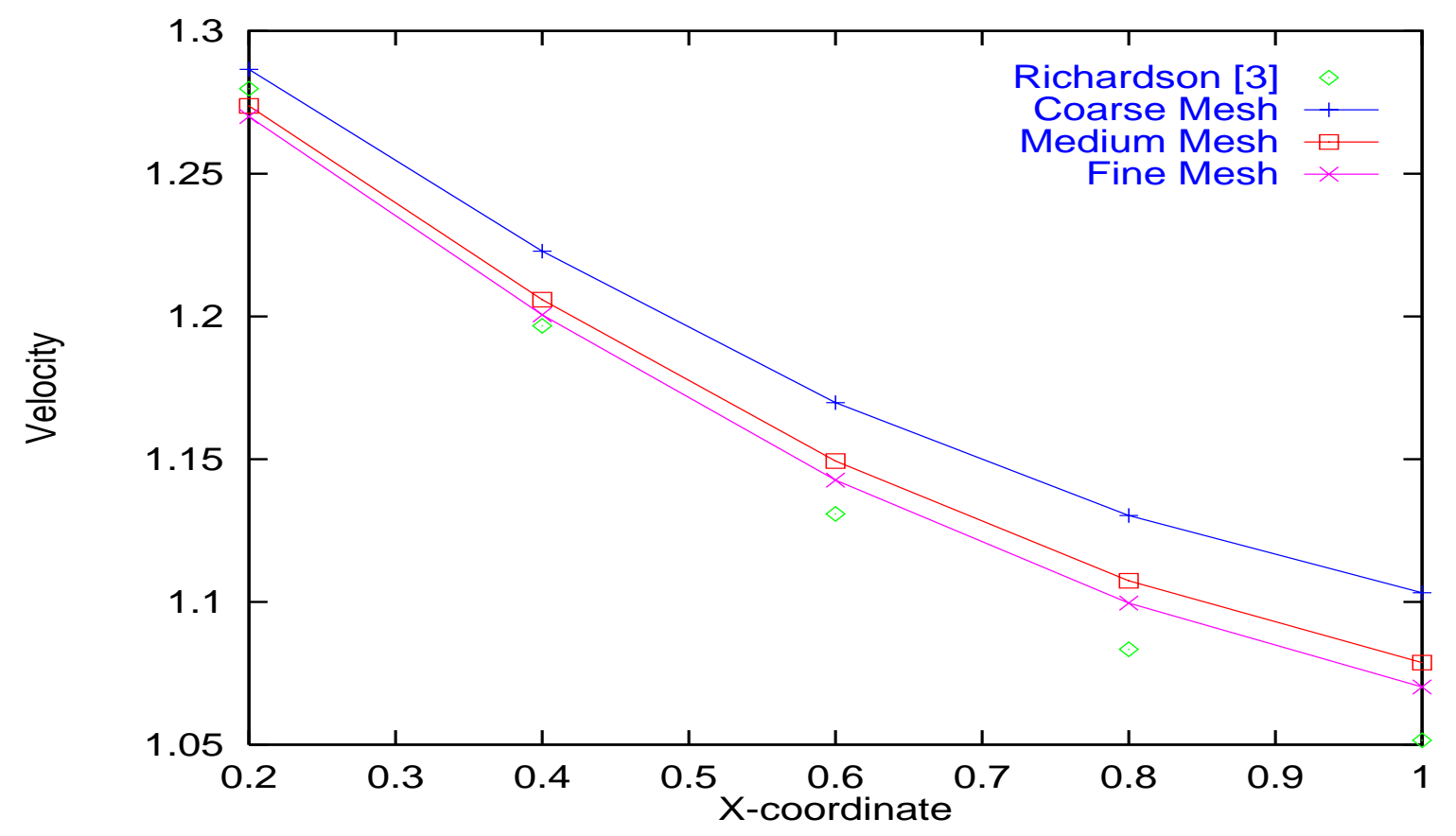

(b) $\mathbf{x}>\mathbf{0}$

Figure 6: Planar stick-slip flow: analytical and numerical solutions for velocity field along centreline $\mathrm{y}=0, \mathrm{Re}=0$ 


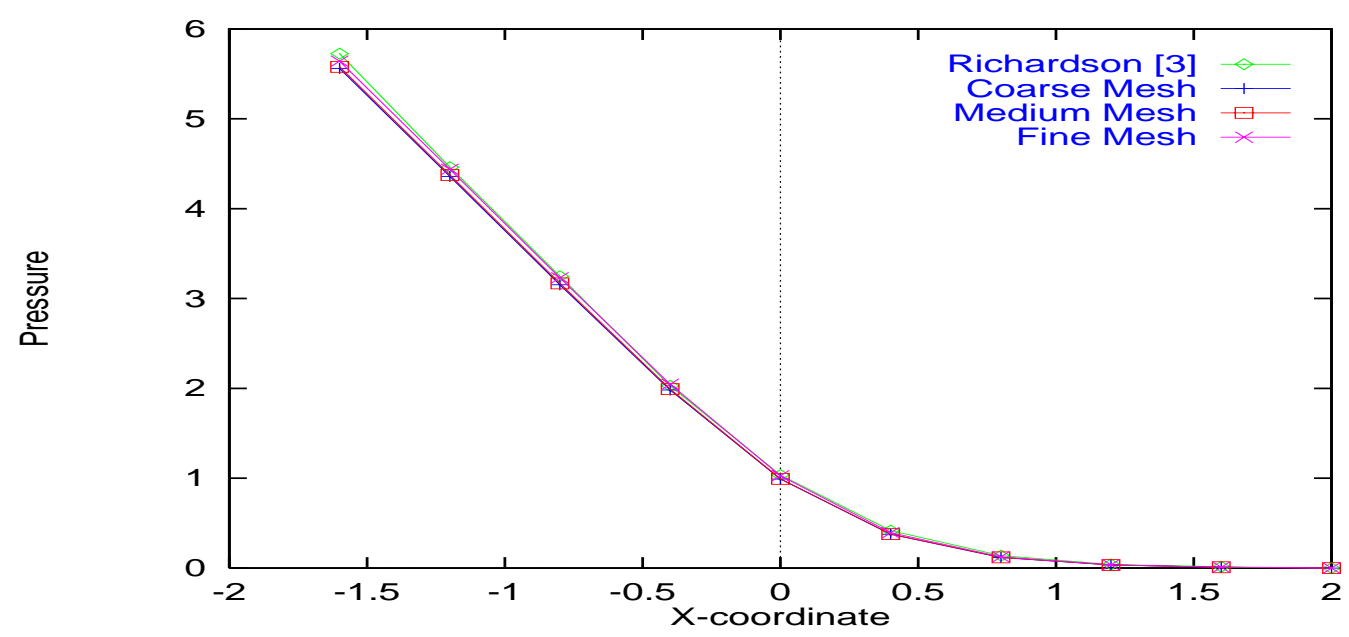

(a) pressure line plot along centreline $\mathbf{y}=0$

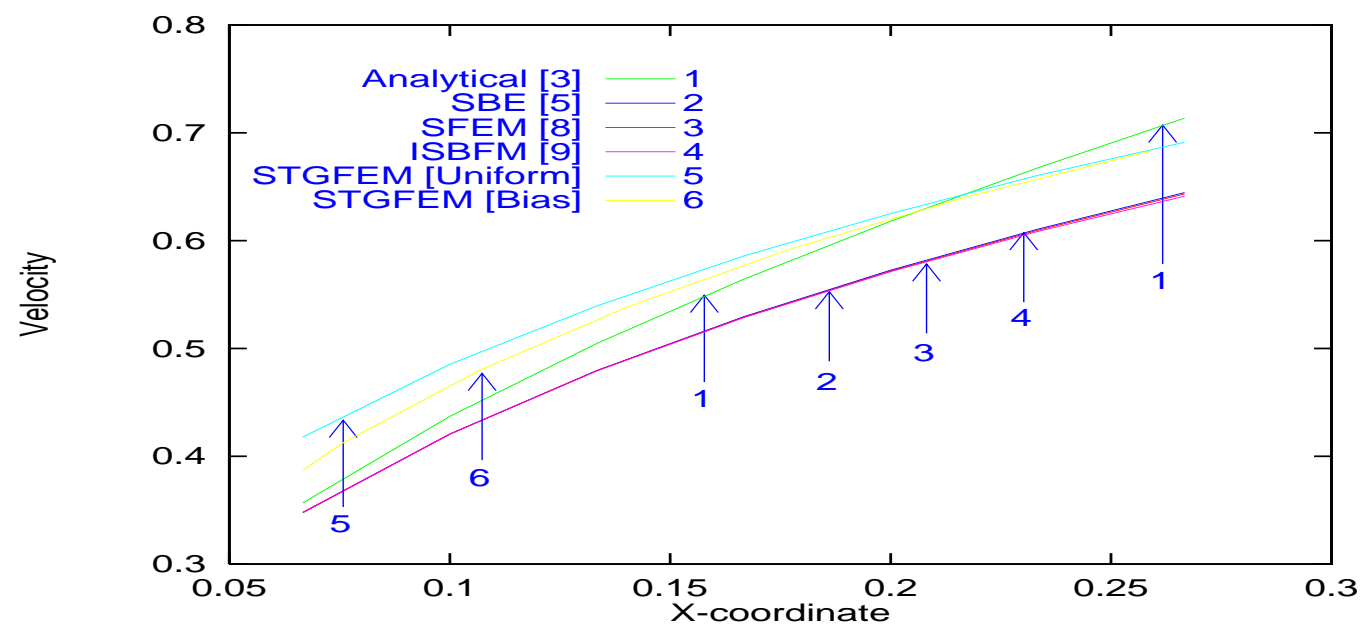

(b) velocity at free surface $(0.05<x<0.30)$

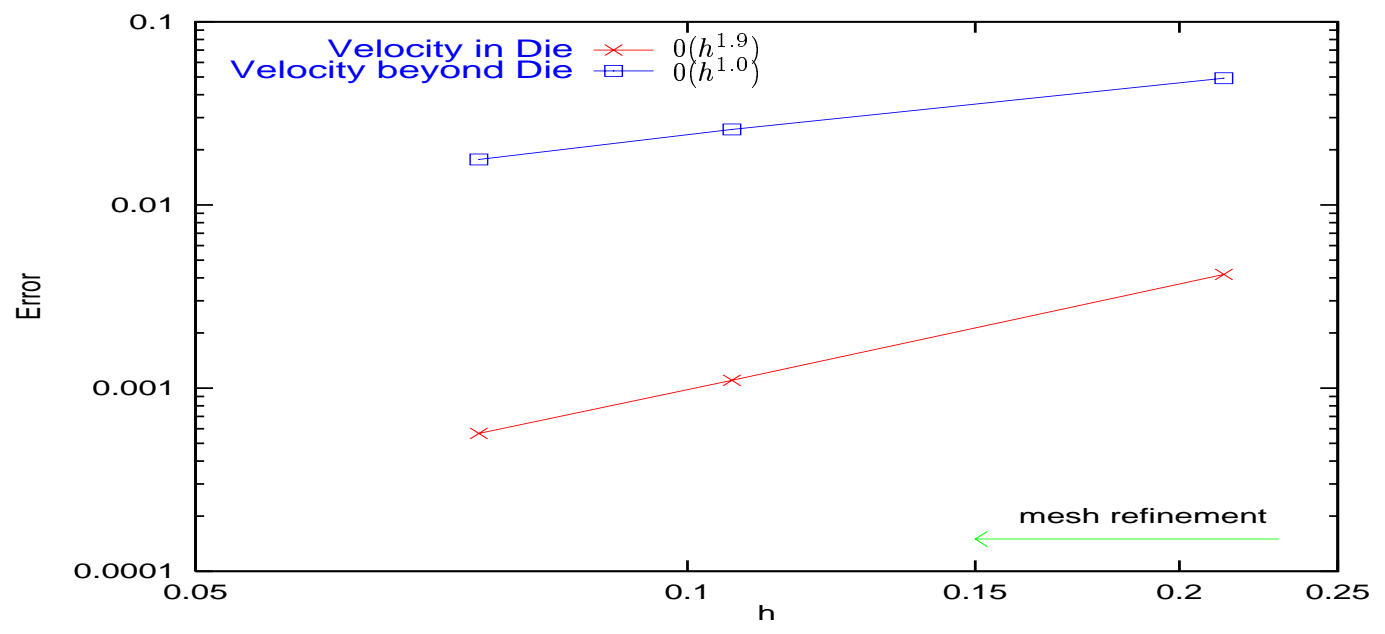

(c) velocity error norms against Richardson [3]

Figure 7: Planar stick-slip flow: analytical and numerical solutions, $R e=0$ 


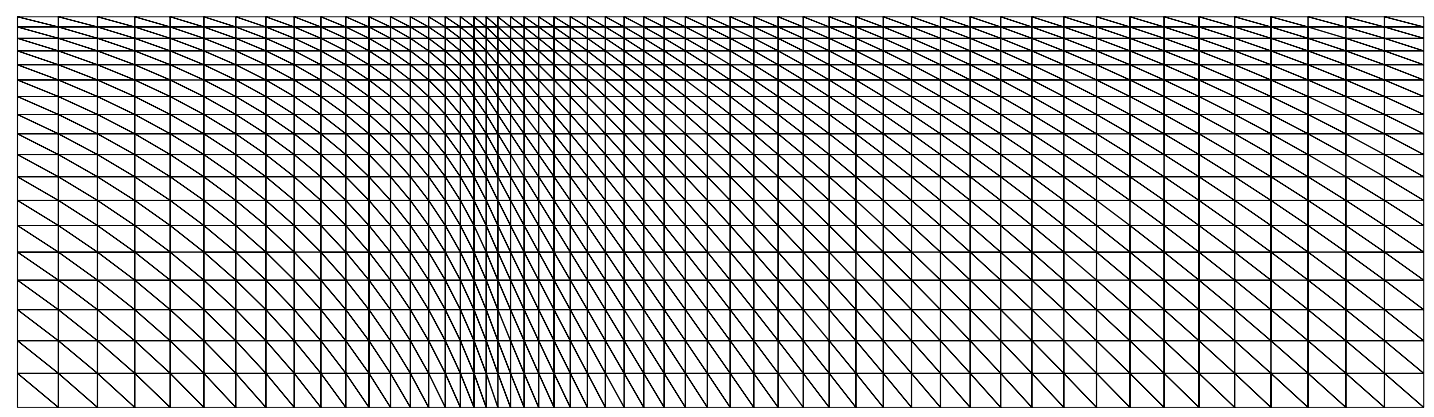

(a) mesh pattern, 18x54 elements

\begin{tabular}{|c|c|c|c|c|c|c|c|c|c|c|c|c|c|c|c|}
\hline - & - & - & - & - & - & - & - & 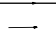 & - & $\longrightarrow$ & 一 & $\longrightarrow$ & $一$ & 一 & 一 \\
\hline - & - & $\stackrel{-}{-}$ & - & $=$ & z & 二 & 二 & 二 & 二 & 二 & 二 & 二 & 二 & 二 & \\
\hline - & - & - & 一 & - & - & - & - & - & - & 二 & 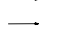 & 二 & 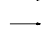 & 二 & \\
\hline 二 & 二 & 二 & $=$ & - & $=$ & - & - & 二 & 一 & 一 & 二 & 二 & 二 & 二 & \\
\hline 二 & $\Rightarrow$ & 二 & 二 & $=$ & 三 & 三 & $\bar{E}$ & 二 & 二 & $\vec{\square}$ & 二 & 二 & 二 & 二 & \\
\hline ? & ? & 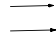 & $=$ & $=$ & $=$ & 二 & 二 & 二 & 二 & 二 & 二 & 二 & 二 & 二 & \\
\hline$\longrightarrow$ & $\longrightarrow$ & $\longrightarrow$ & $\longrightarrow$ & $\longrightarrow$ & $\longrightarrow$ & 一 & 一 & 一 & 一 & 一 & 一 & 一 & 二 & 二 & \\
\hline 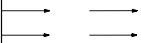 & 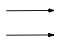 & ? & ? & ב & $=$ & 二 & 二 & 二 & 二 & 二 & 二 & 二 & 二 & 二 & \\
\hline$\longrightarrow$ & $\longrightarrow$ & $\longrightarrow$ & $\longrightarrow$ & $\longrightarrow$ & 二 & 二 & 二 & 一 & 一 & 二 & 二 & 二 & 二 & 二 & \\
\hline$\longrightarrow$ & $\longrightarrow$ & $\longrightarrow$ & $\longrightarrow$ & $\longrightarrow$ & 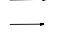 & $\Longrightarrow$ & 二 & $\Rightarrow$ & 一 & 二 & 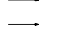 & 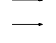 & 二 & 二 & \\
\hline
\end{tabular}

(b) velocity vectors
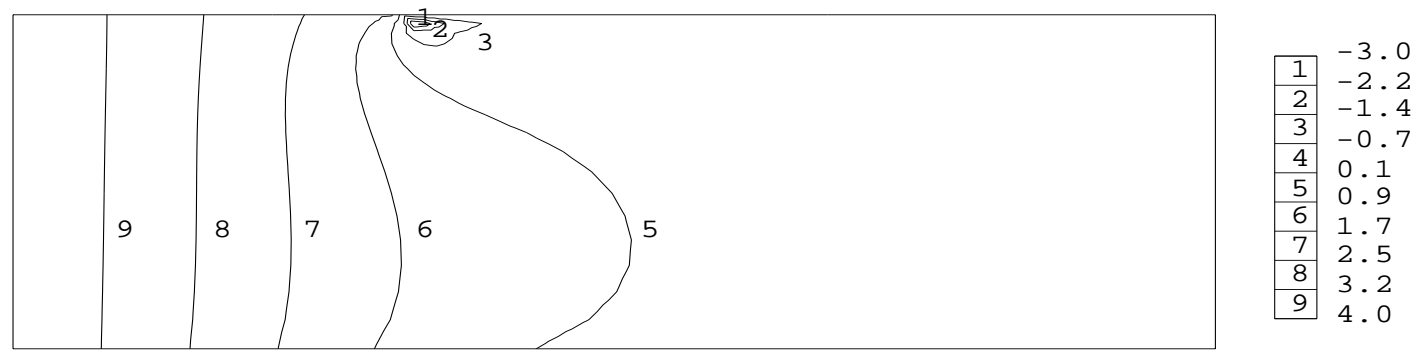

(c) pressure contours
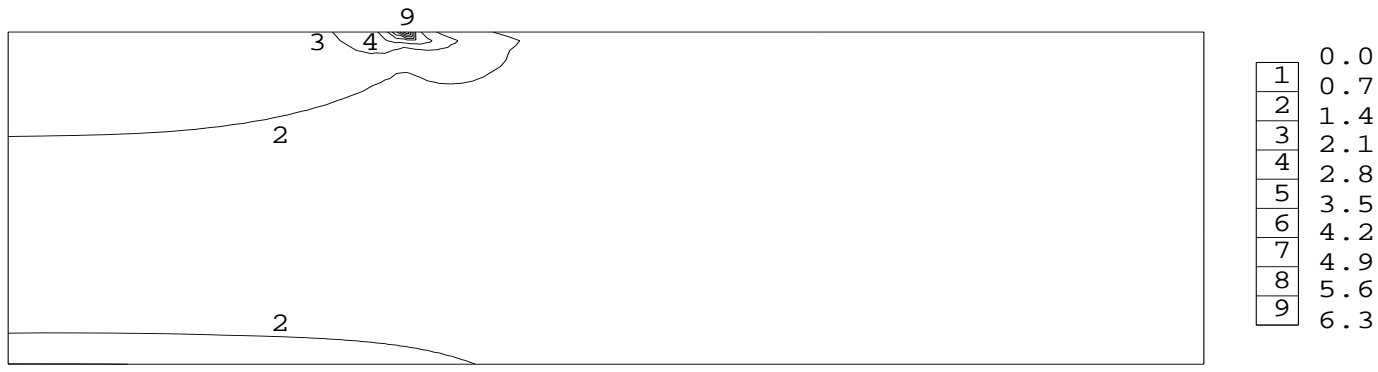

(d) $I_{2}$ contours

Figure 8: Stick-slip/drag flow: $\mathbf{R e}=10^{-4}$ 


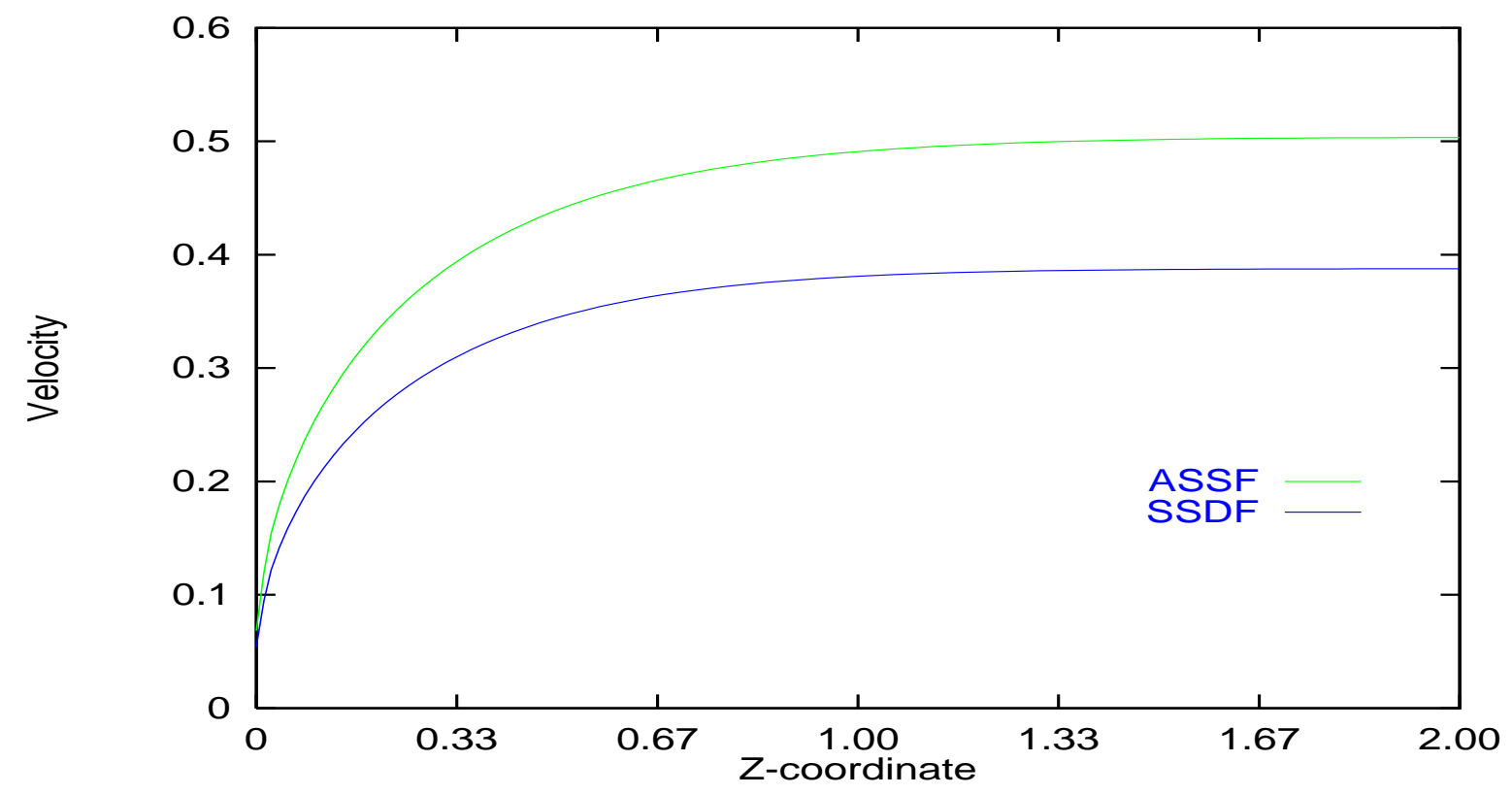

(a) $V_{z}$ on free surface

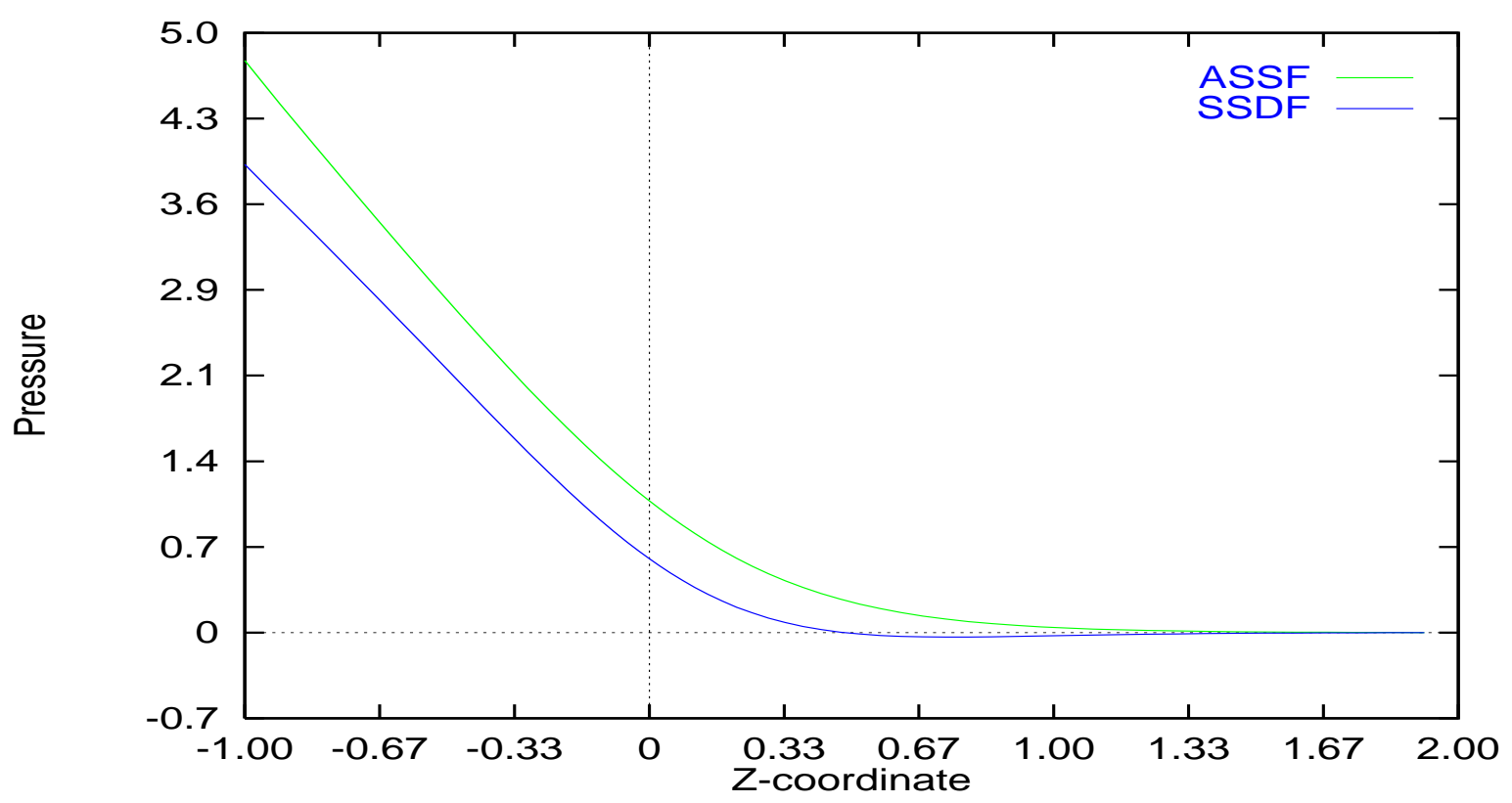

(b) pressure on axis of symmetry for stick-slip flow and inner surface for stick-slip/drag flow

Figure 9: Stick-slip flow and stick-slip/drag flow, Re $=10^{-4}$ 


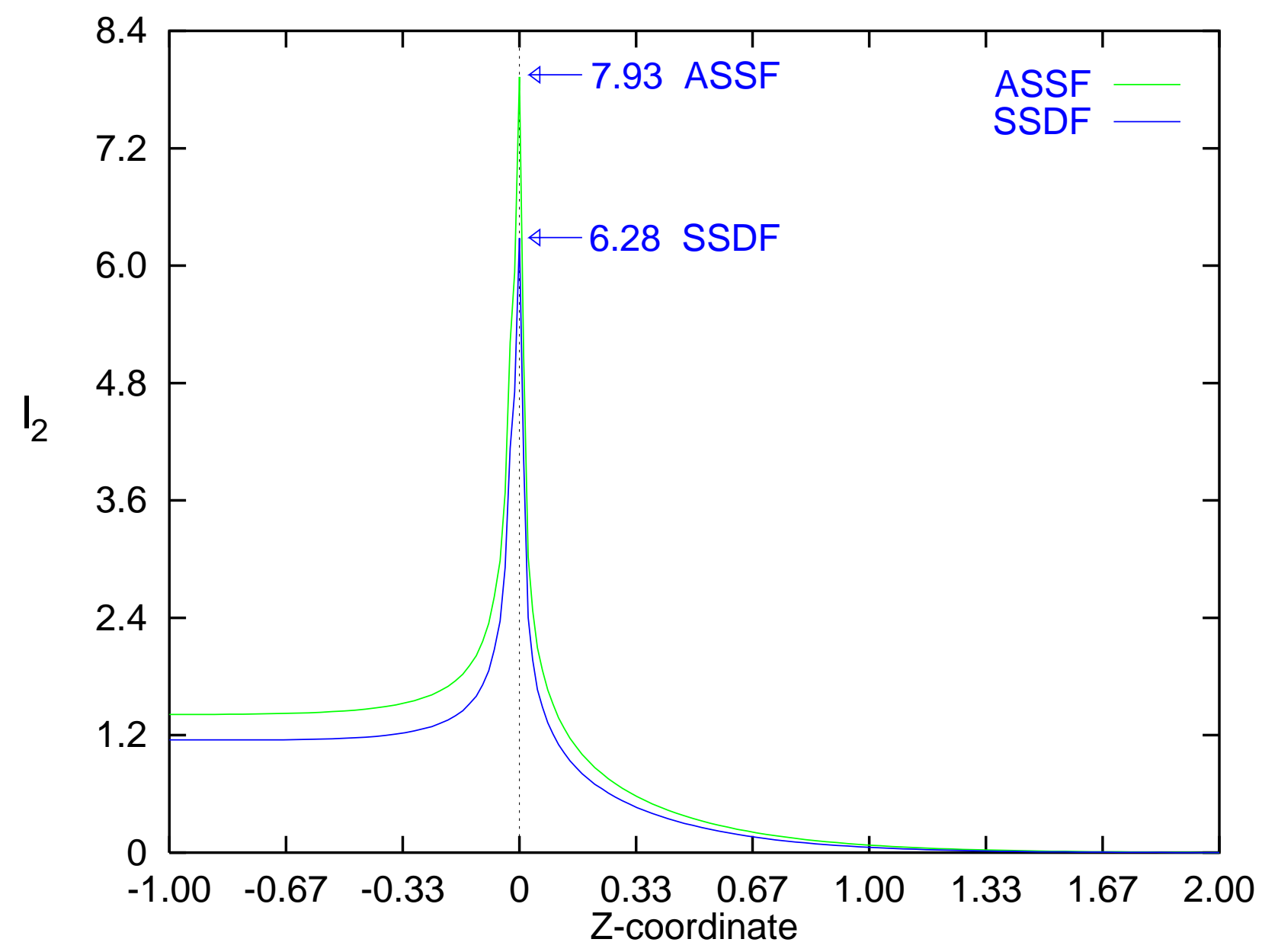

(c) $I_{2}$ on free surface

Figure 9: Continued Stick-slip flow and stick-slip/drag flow, $\mathbf{R e}=10^{-4}$ 


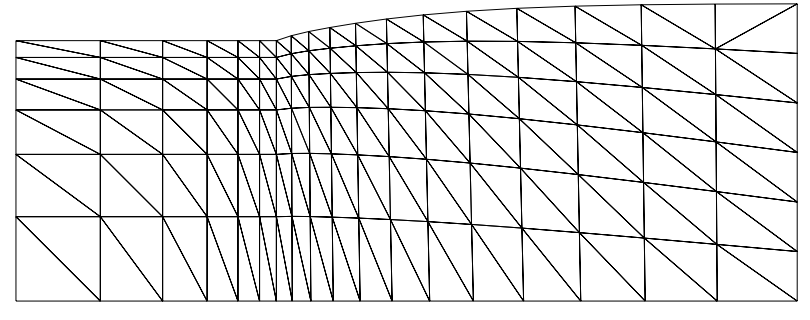

(a) coarse UD mesh, $6 \times 18$ elements

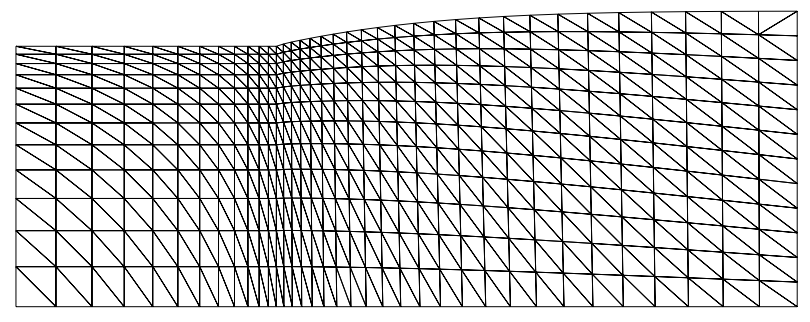

(b) medium UD mesh, 12x36 elements

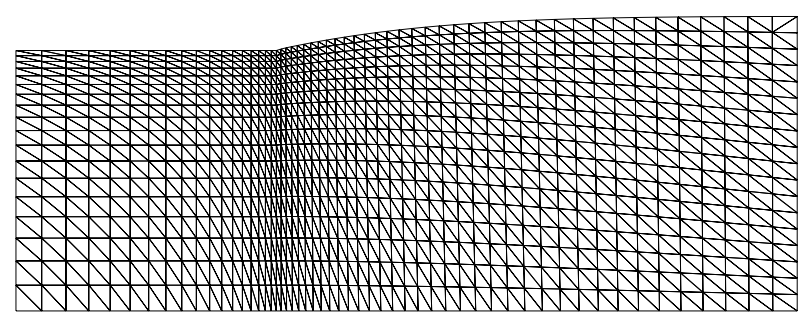

(c) fine UD mesh, 18x54 elements

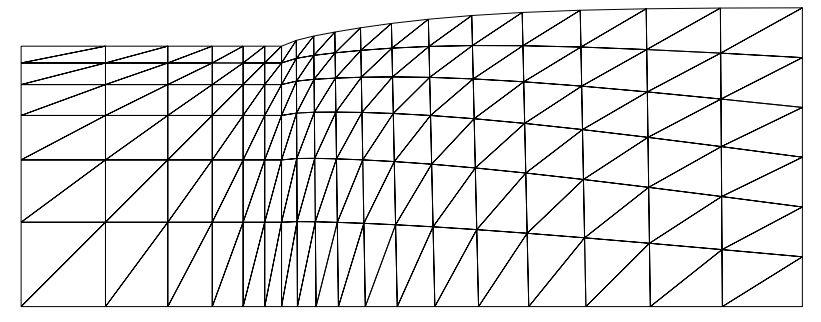

(d) coarse DU mesh, 6x18 elements

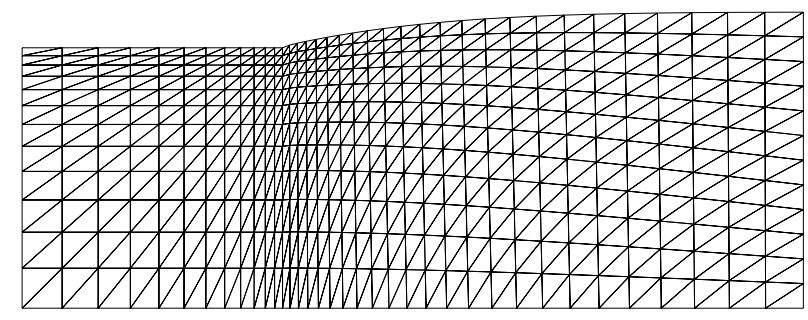

(e) medium DU mesh, 12x36 elements

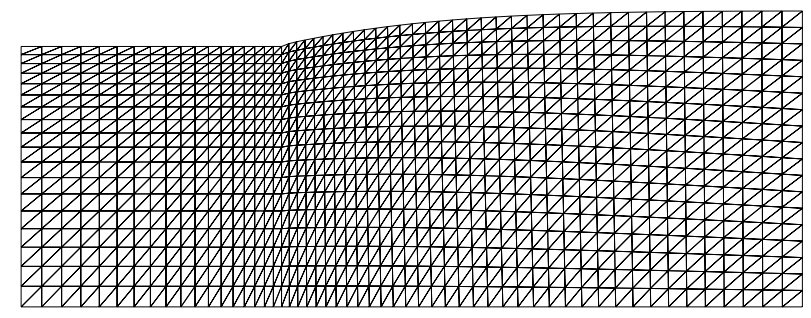

(f) fine DU mesh, 18x54 elements

Figure 10: Die-swell flow: mesh patterns 


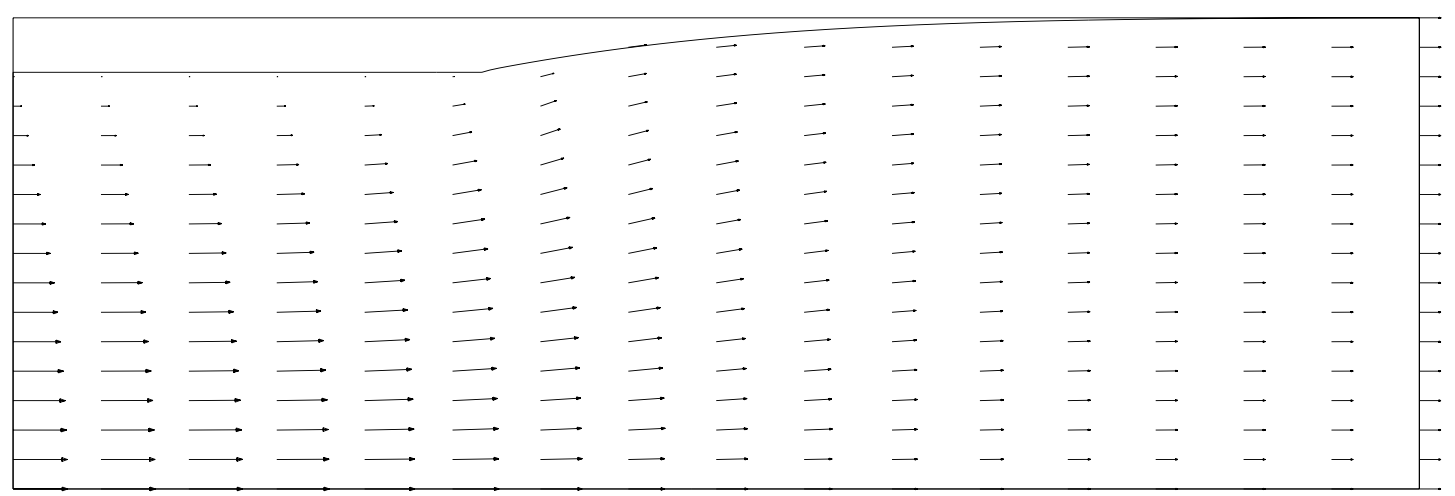

(a) velocity vectors

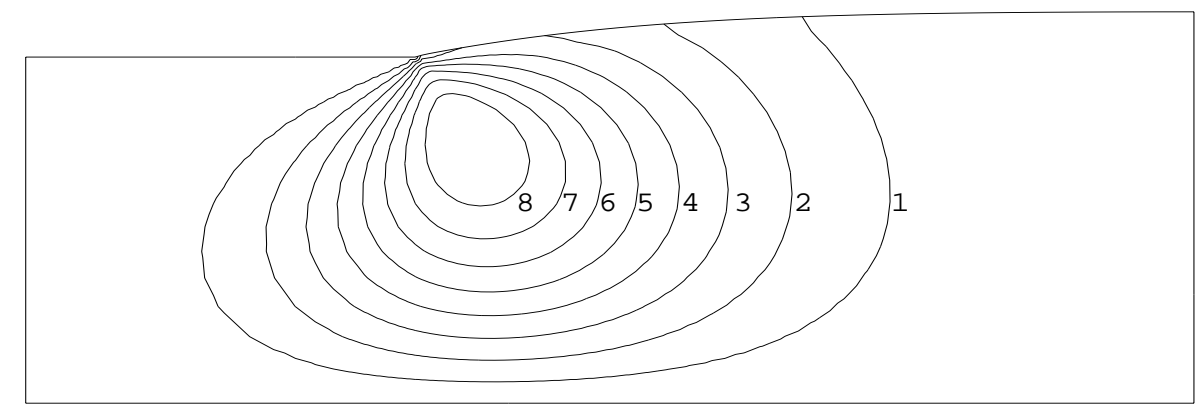

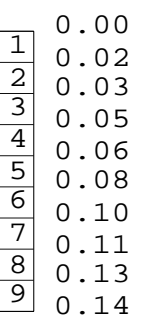

(b) $V_{r}$ contours

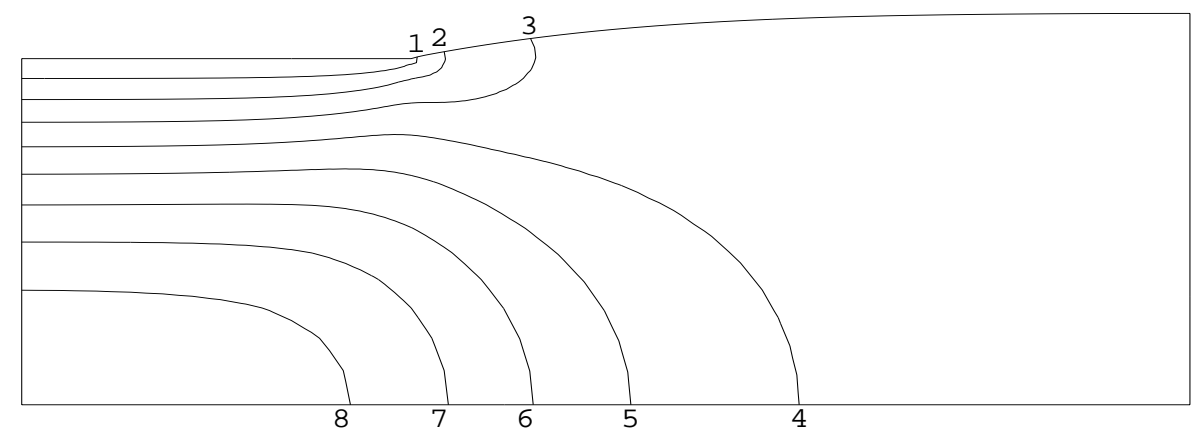

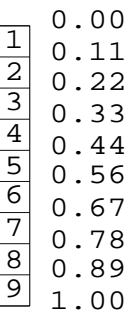

(c) $V_{z}$ contours

Figure 11: Die-swell flow: fine UD mesh, $\mathbf{R e}=10^{-4}$ 


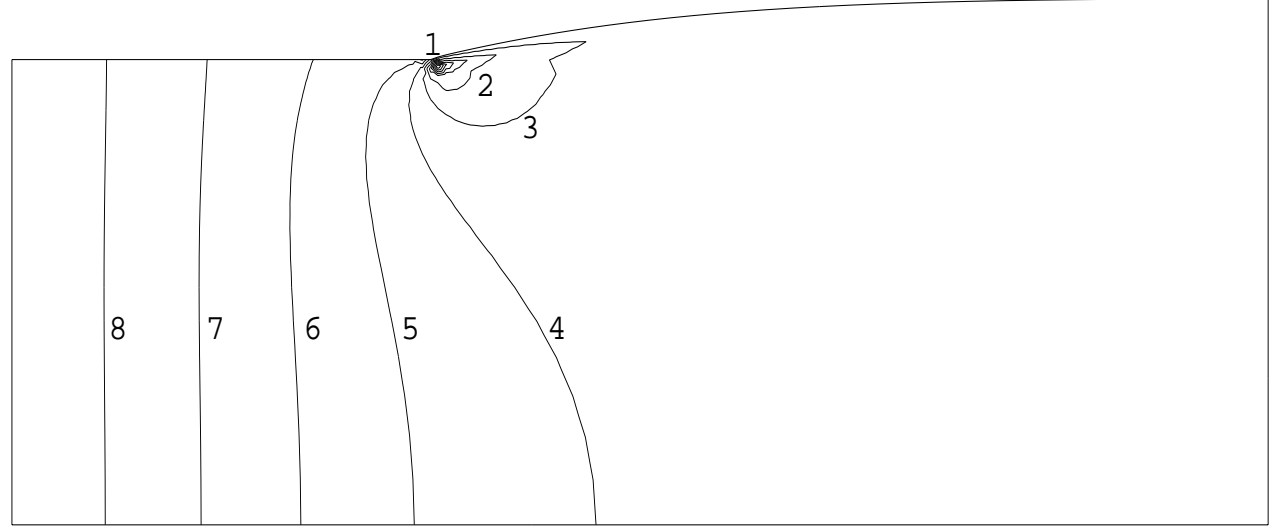

$-7.1$

$1-5.8$

$2-4.4$

$3-3.1$

$4-1.7$

$5-0.4$

61.0

72.3

8.6

$9 \quad 4.9$

(d) pressure contours

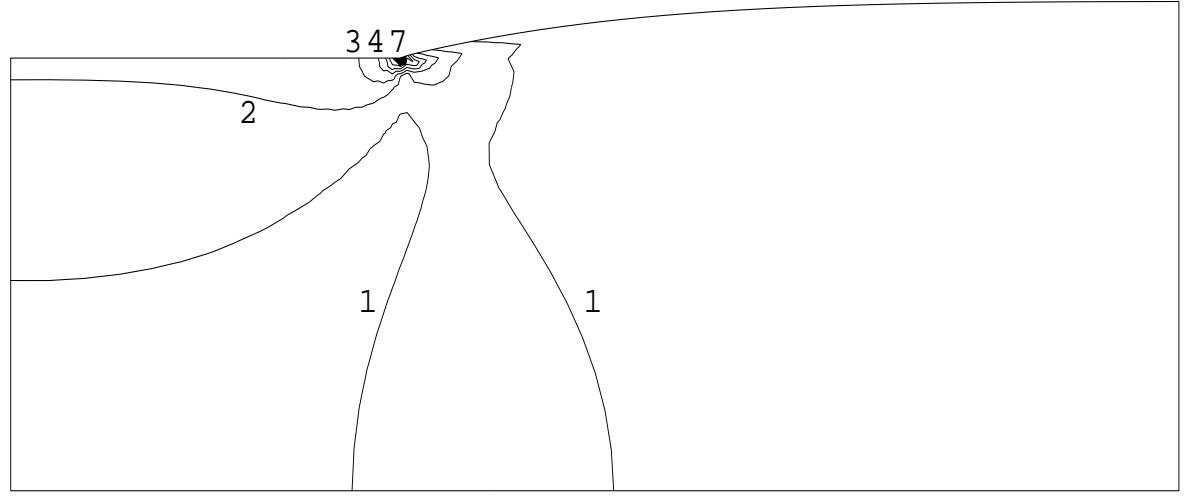

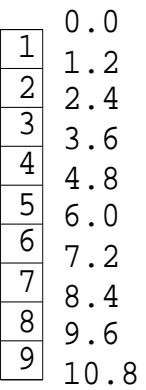

(e) $I_{2}$ contours

Figure 11: Continued Die-swell flow: fine UD mesh, $\mathbf{R e}=10^{-4}$ 


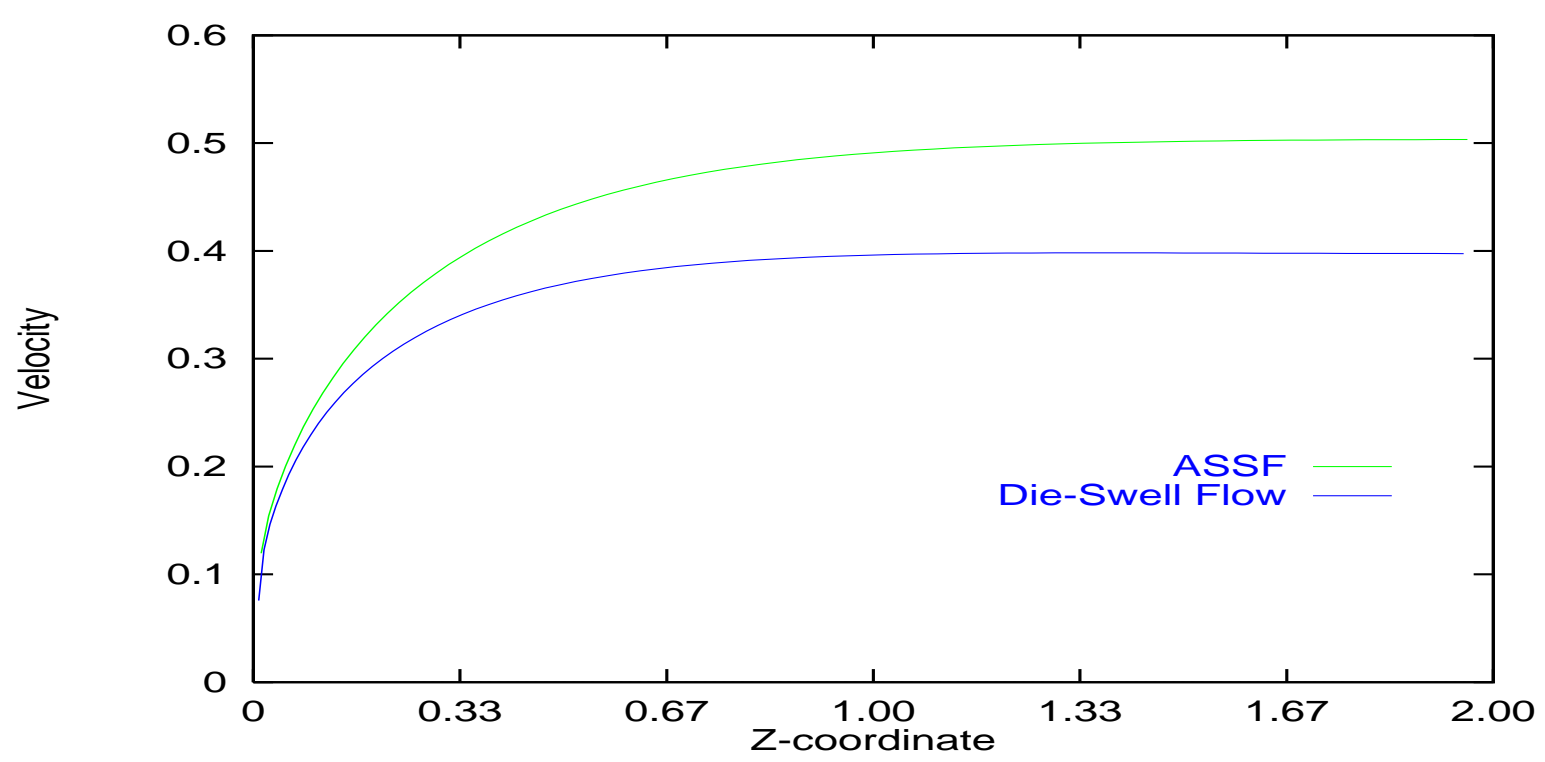

(a) $V_{z}$ on free surface

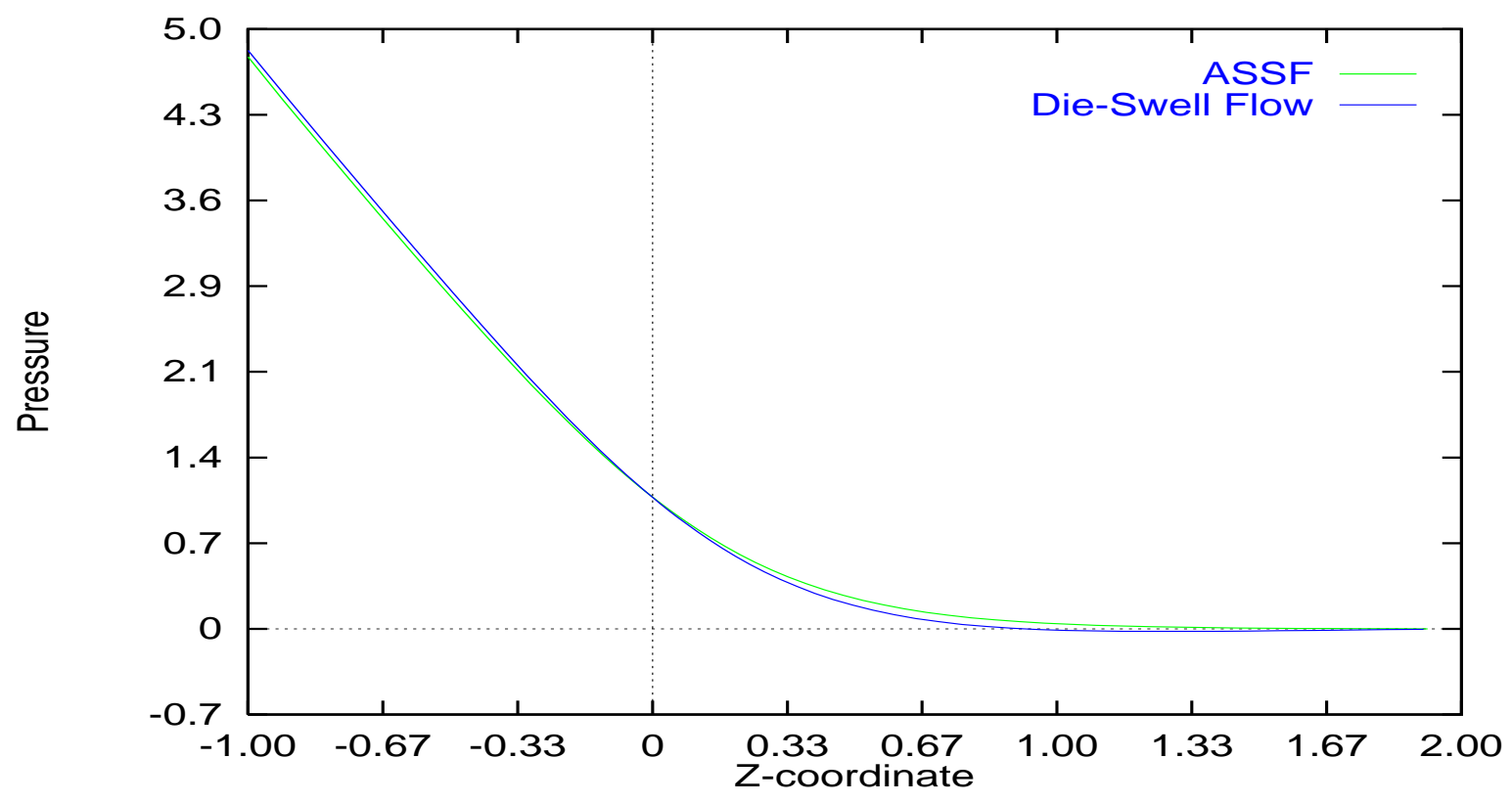

(b) pressure on axis of symmetry for stick-slip flow and die-swell flow

Figure 12: Stick-slip flow and die-swell flow, fine UD mesh, $R e=10^{-4}$ 


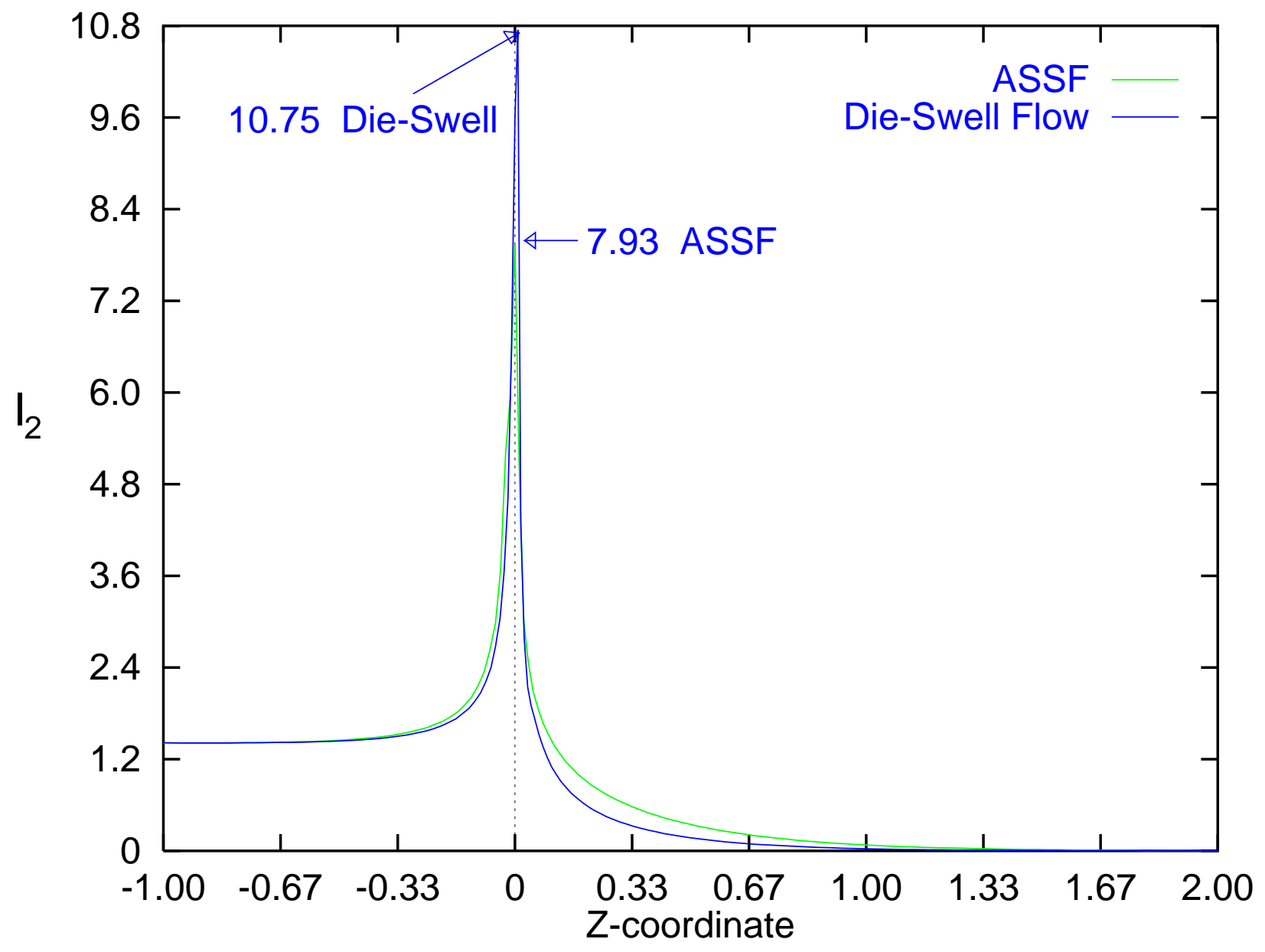

(c) $I_{2}$ on free surface

Figure 12: Continued Stick-slip flow and die-swell flow, fine UD mesh, $R e=10^{-4}$ 


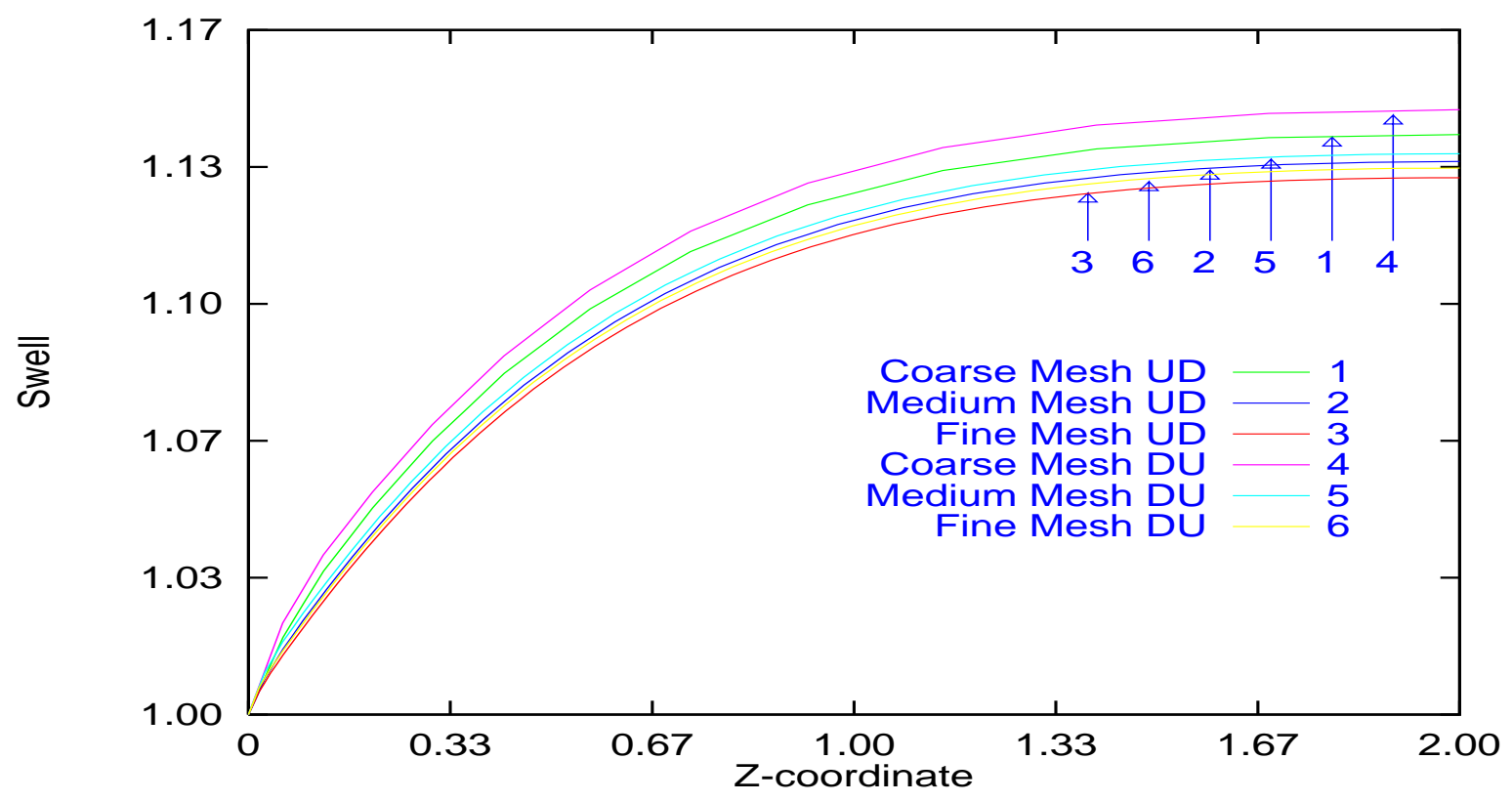

(a) swell free surface with mesh refinement

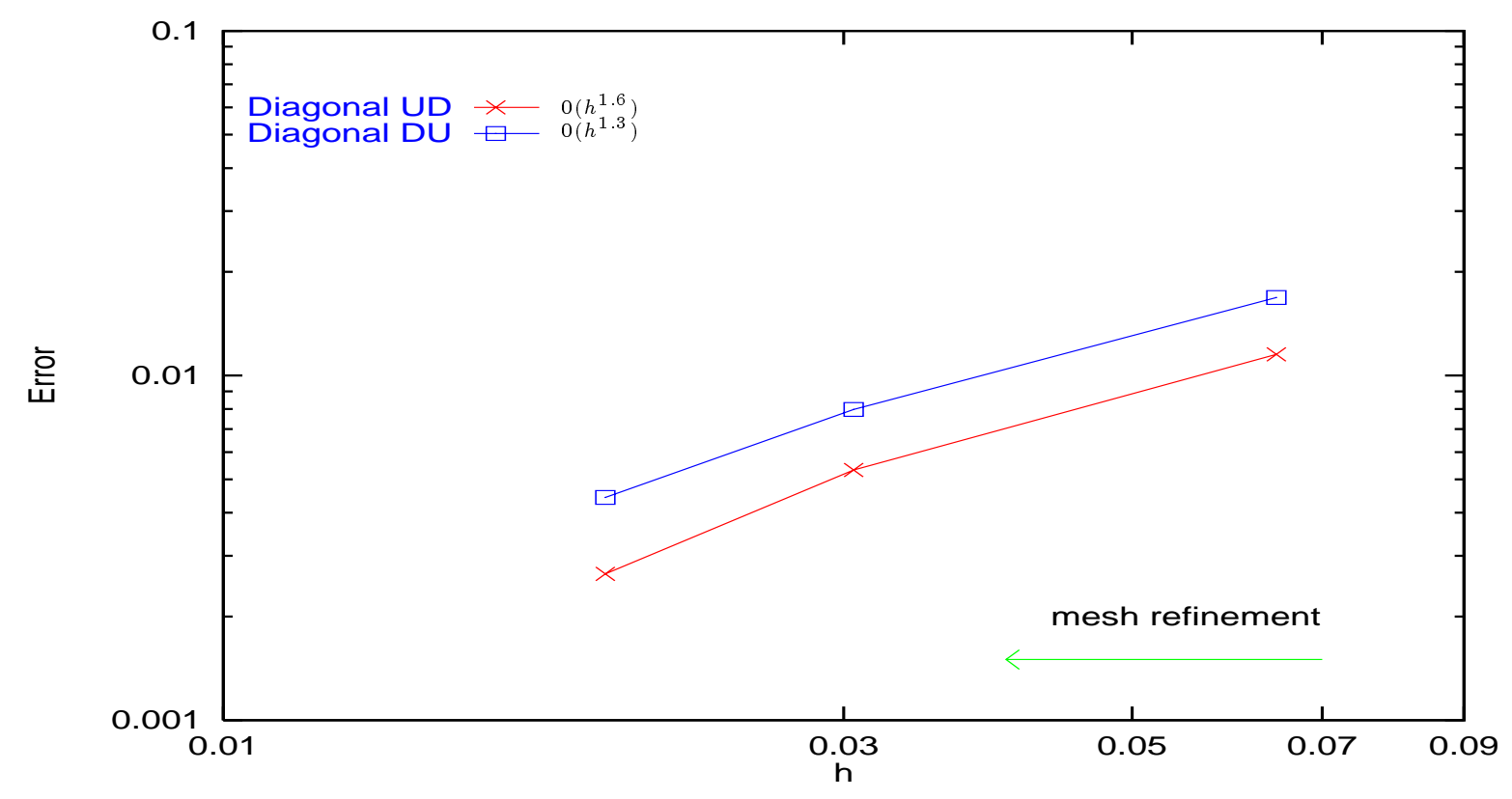

(b) die-swell error norms against Nickell et al. [11]

Figure 13: Die-swell flow: comparison of solutions, $\mathbf{R e}=10^{-4}$ 

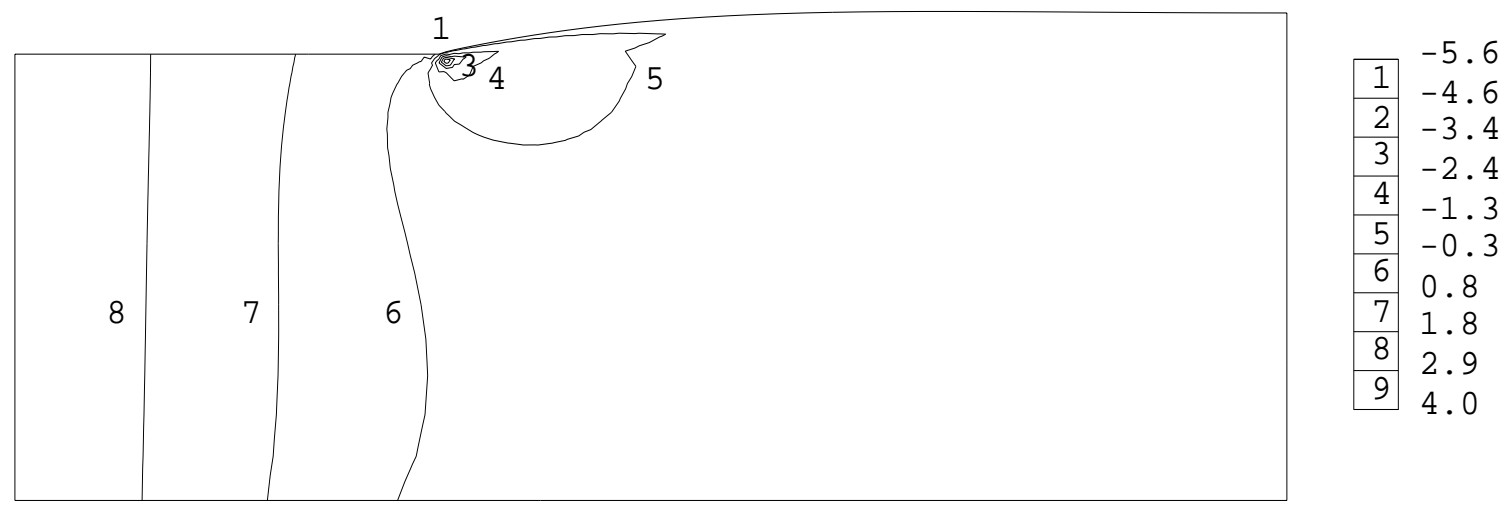

(a) pressure contours

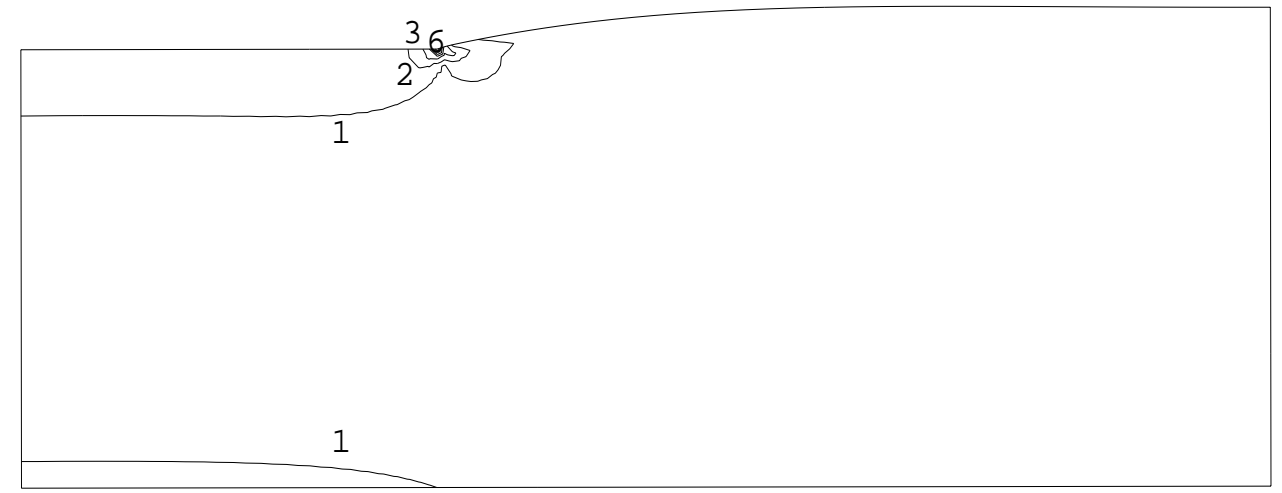

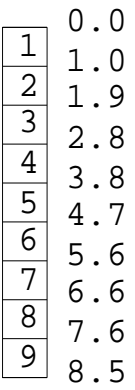

(b) $I_{2}$ contours

Figure 14: Die-swell/drag flow: fine UD mesh, $\mathbf{R e}=10^{-4}$ 


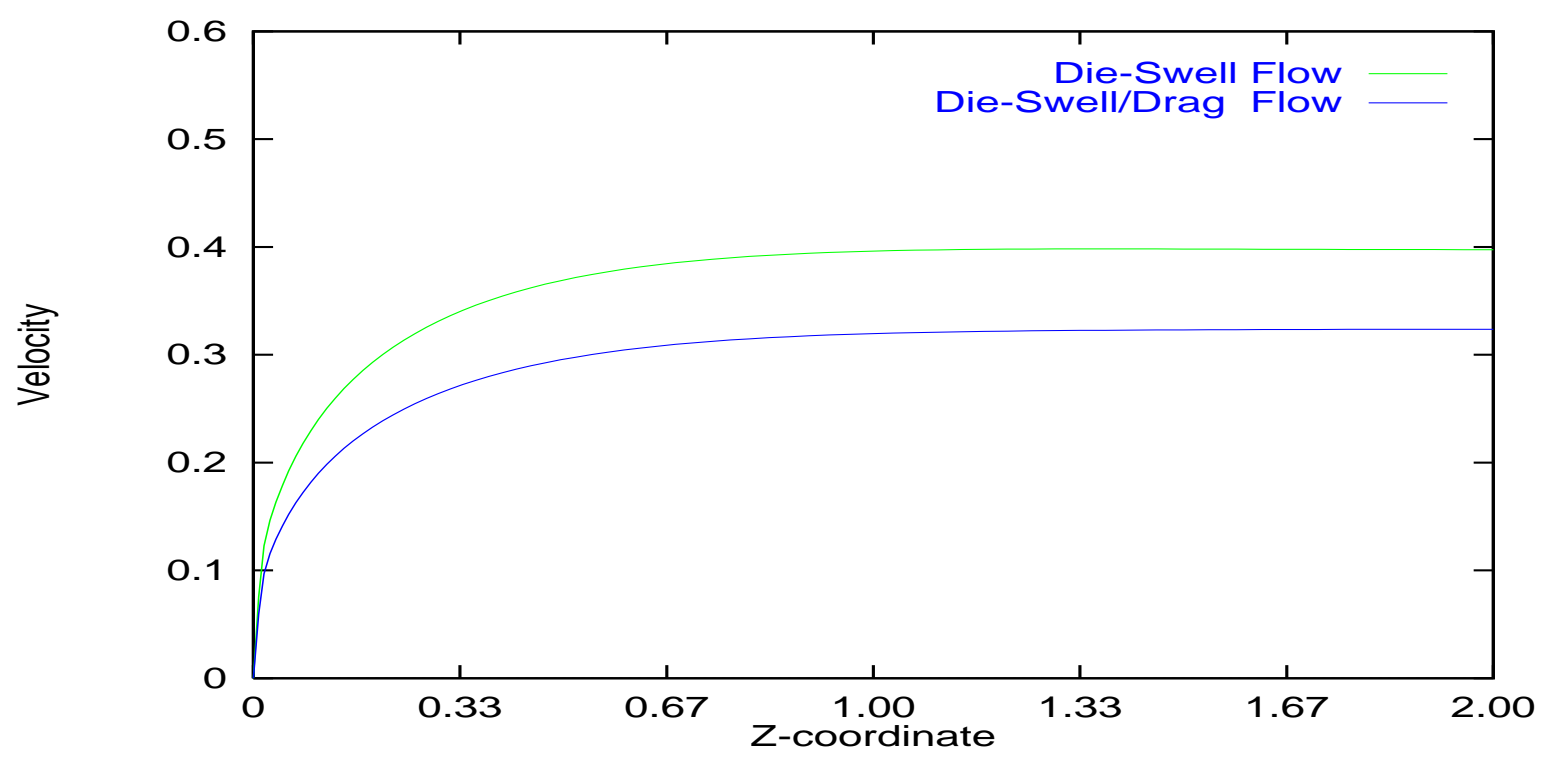

(a) velocity

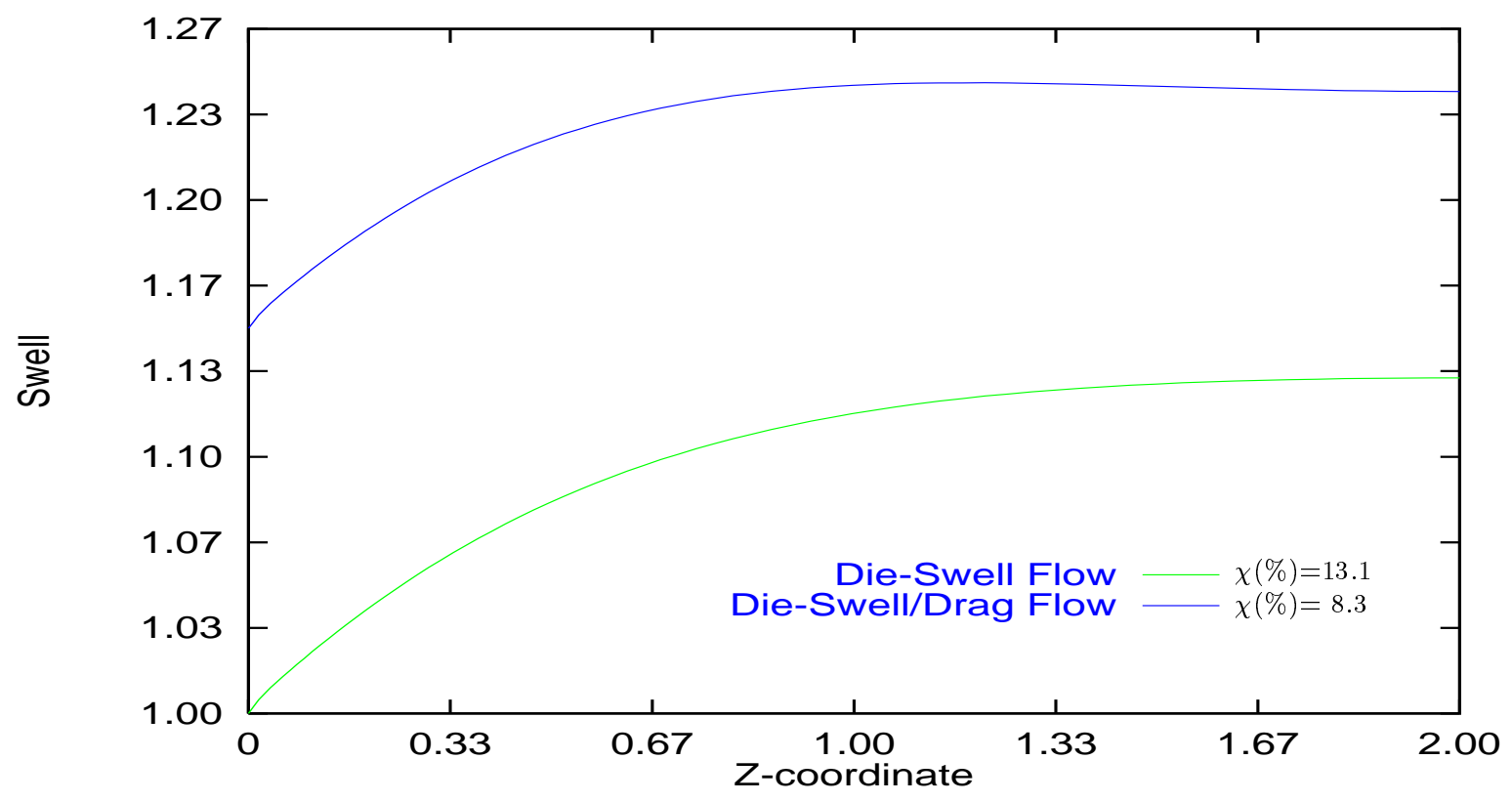

(b) die swell

Figure 15: Die-swell flow and die-swell/drag flow, fine UD mesh, Re $=10^{-4}$ 


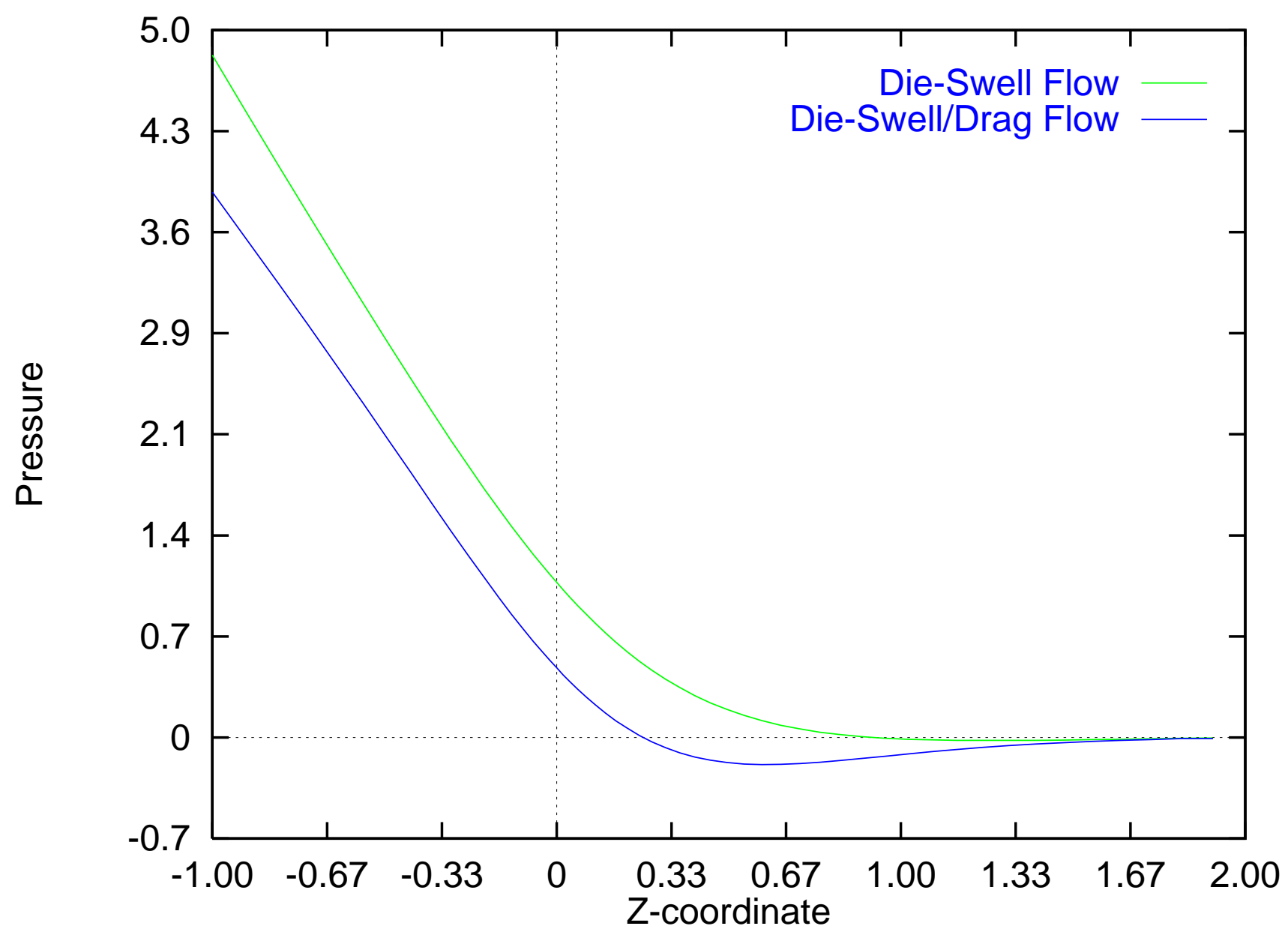

(c) pressure on axis of symmetry for die-swell flow and inner surface for die-swell/drag flow

Figure 15: Continued Die-swell flow and die-swell/drag flow, fine UD mesh, $\boldsymbol{R e}=10^{-4}$ 


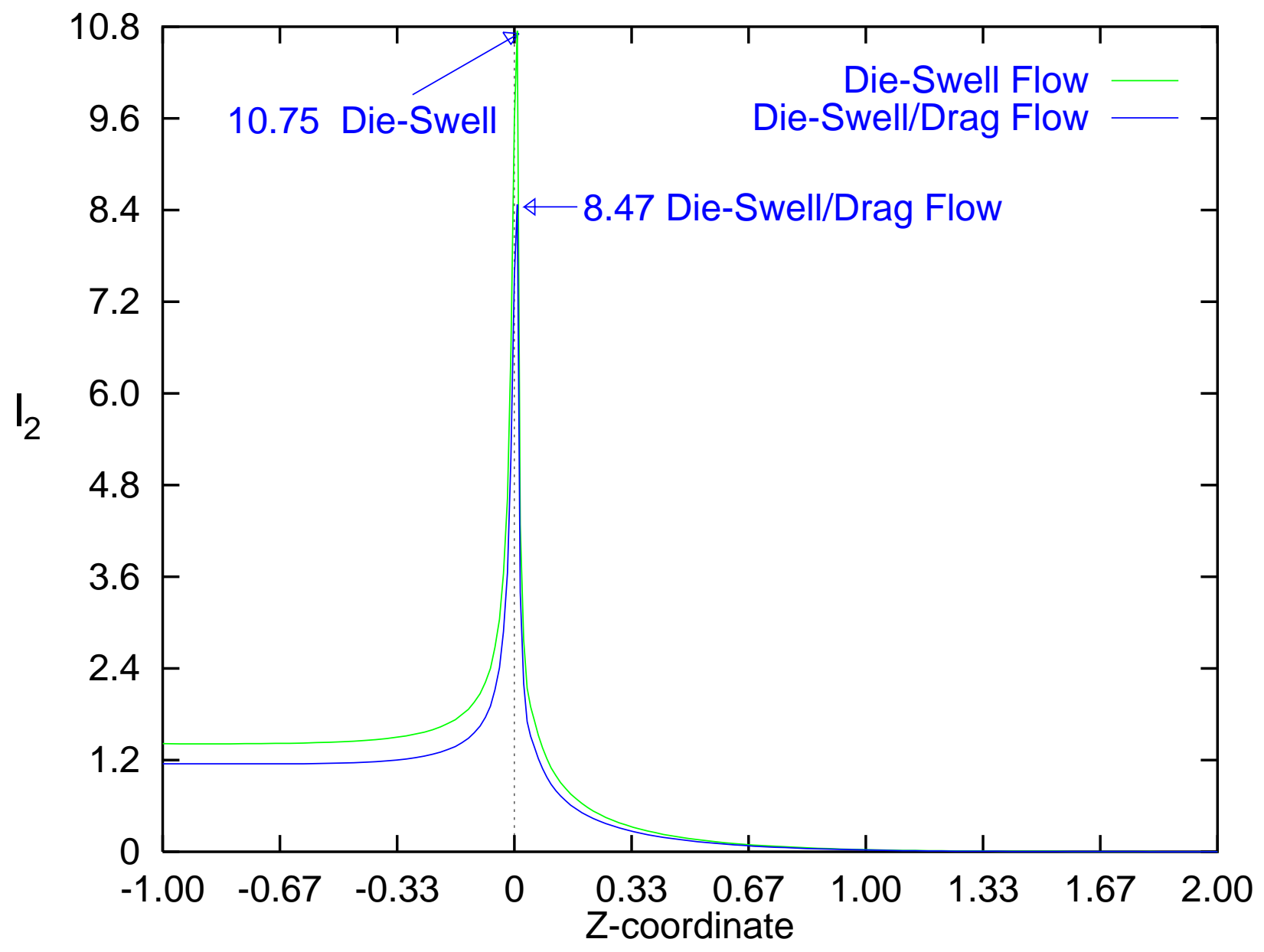

(d) $I_{2}$ on free surface

Figure 15: Continued Die-swell flow and die-swell/drag flow, fine UD mesh, $\boldsymbol{R e}=10^{-4}$ 